\title{
On the continuity of the finite Bloch-Kato cohomology
}

\author{
$\operatorname{AdRiAn~IOVITA}(*)$ - AdRIANo Marmora $(* *)$
}

ABSTRACT - Let $K_{0}$ be an unramified, complete discrete valuation field of mixed characteristics $(0, p)$ with perfect residue field. We consider two finite, free $Z_{p}$-representations of $G_{K_{0}}, T_{1}$ and $T_{2}$, such that $T_{i} \otimes z_{p} Q_{p}$, for $i=1,2$, are crystalline representations with Hodge-Tate weights between 0 and $r \leq p-2$. Let $K$ be a totally ramified extension of degree $e$ of $K_{0}$. Supposing that $p \geq 3$ and $e(r-1) \leq p-1$, we prove that for every integer $n \geq 1$ and $i=1,2$, the inclusion $H_{f}^{1}\left(K, T_{i}\right) / p^{n} H_{f}^{1}\left(K, T_{i}\right) \hookrightarrow H^{1}\left(K, T_{i} / p^{n} T_{i}\right)$ of the finite Bloch-Kato cohomology into the Galois cohomology is functorial with respect to morphisms as $Z_{1} / p^{n} Z_{[}\left[G_{K_{0}}\right]$-modules from $T_{1} / p^{n} T_{1}$ to $T_{2} / p^{n} T_{2}$. In the appendix we give a related result for $p=2$.

Mathematics SubJect Classification (2010). 11S32 ; 14F30

KEYworDs. Selmer groups, Galois representations, lattices in crystalline representations, Bloch-Kato finite cohomology, strongly divisible modules.

\section{Contents}

1. Introduction .......................................... 240

1.1 The main result ...................................... 242

1.2 Notation and conventions ............................... 245

2. Strongly divisible modules ............................... 245

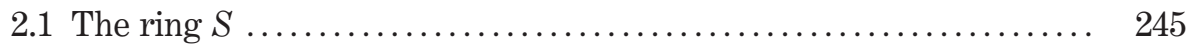

2.2 Strongly divisible lattices over $S \ldots \ldots \ldots \ldots \ldots \ldots \ldots \ldots \ldots \ldots, 246$

2.3 Functors towards Galois representations ................. 247

(*) Indirizzo dell'A.: Department of Mathematics and Statistics, Concordia University, 1455 de Maisonneuve Blvd. West, Montréal, Québec H3G 1M8, Canada, and Dipartimento di Matematica Pura ed Applicata, Università degli Studi di Padova, Via Trieste 63, 35121 Padova, Italy.

E-mail: iovita@mathstat.concordia.ca

This author was partly funded by the grant PRIN-MIUR 2010-11, "Arithmetic Algebraic Geometry and Number Theory" and an NSERC Discovery grant.

(**) Indirizzo dell'A.: IRMA, Université de Strasbourg et CNRS, 7 rue René-Descartes, 67084 Strasbourg, France.

E-mail: marmora@math.unistra.fr

This author was partly supported by the project ANR-09-JCJC-0048-01. 
3. Extensions of crystalline representations .................... 250

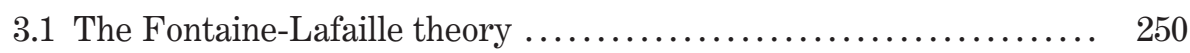

3.2 Relation with strongly divisible lattices over $S \ldots \ldots \ldots \ldots \ldots \ldots . . \ldots 252$

3.3 A double complex computing extensions .................... 254

4. Proof of the main result.................................... 259

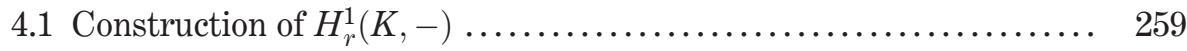

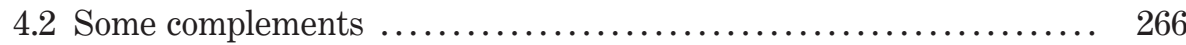

A. The characteristic two case $\ldots \ldots \ldots \ldots \ldots \ldots \ldots \ldots \ldots \ldots \ldots \ldots . . \ldots, 268$

\section{Introduction}

Let $K$ denote a complete discrete valuation field of mixed characteristics $(0, p)$ with perfect residue field $k$. We choose an algebraic closure $\bar{K}$ of $K$ and denote by $G_{K}$ the Galois group of $\bar{K}$ over $K$. For a $p$-adic representation $V$ of $G_{K}$, the finite cohomology group $H_{f}^{1}(K, V)$ is defined as

$$
H_{f}^{1}(K, V)=\operatorname{ker}\left(H^{1}(K, V) \rightarrow H^{1}\left(K, V \otimes_{Q_{p}} \mathrm{~B}_{\text {cris }}\right)\right)
$$

where $H^{1}(K,-)$ denotes the first continuous Galois cohomology functor and $\mathrm{B}_{\text {cris }}$ is the ring of crystalline periods. If $V$ is a crystalline representation then the cohomology classes in $H_{f}^{1}(K, V)$ represent classes of extensions

$$
0 \rightarrow V \rightarrow E \rightarrow Q_{p} \rightarrow 0
$$

which are crystalline, i.e. such that $E$ is a crystalline $G_{K}$-representation. If $T \subset V$ is a $Z_{p}$-lattice stable by $G_{K}$, the group $H_{f}^{1}(K, T)$ is defined as the fiber product of the diagram

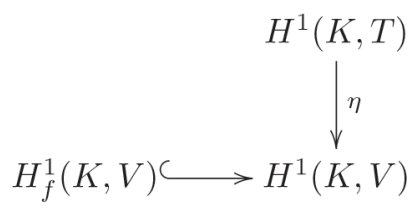

where $\eta$ is the map induced by the injection $T \subset V$. By definition the classes in $H_{f}^{1}(K, T)$ correspond to classes of extensions of $T$ by $Z_{p}$ which are crystalline (after extending scalars to $\left.Q_{p}\right)$. The canonical map $H_{f}^{1}(K, T) \rightarrow H^{1}(K, T)$ is injective and identifies $H_{f}^{1}(K, T)$ to $\eta^{-1}\left(H_{f}^{1}(K, V)\right)$; on the other hand the map $H_{f}^{1}(K, T) \rightarrow$ $H_{f}^{1}(K, V)$ is not injective in general $\left(H_{f}^{1}(K, T)\right.$ contains all the $p$-torsion of $\left.H^{1}(K, T)\right)$.

The main relevance of the groups $H_{f}^{1}(K, V)$ comes from global arithmetic. Suppose that $F$ is a number field and that $M$ is a $p$-adic representation of $G_{F}:=\operatorname{Gal}(\bar{F} / F)$. Then the Selmer group of $M$ over $F$ is the subgroup of the continuous cohomology group $H^{1}(F, M)$ of classes which satisfy certain local restrictions, in particular the classes in the Selmer group restricted to the decomposition group at $v$ of $G_{F}$, for places $v$ of $F$ dividing $p$, lie in $H_{f}^{1}\left(F_{v}, M\right)$. 
If the $p$-adic representation $M$ as above is the $p$-adic realization of a motive over a number field $F$, then the family of $p$-Selmer groups over the cyclotomic tower of $F$ (or some other $Z_{p}$-tower) encodes important arithmetic information about the motive, in particular its algebraic $p$-adic $L$-functions are defined in terms of it. Ralph Greenberg was the first to ask the question: suppose we have two motives which are congruent modulo a power of $p$. What can one say about the two families of $p$-Selmer groups?

For example in [14] and [13] one studies the case of two elliptic curves defined over $Q$ (or even modular forms of weight two) and one obtains: if the elliptic curves are both ordinary or both supersingular at $p$ and they are congruent modulo $p$, then under certain further hypothesis one shows that the families of non-primitive Selmer groups attached to these elliptic curves are also congruent modulo $p$. It follows, using results of $\mathrm{K}$. Kato that if the main conjecture holds for one of the elliptic curves then it holds for the other.

Of course in order to study the behaviour of Selmer groups with respect to congruences, one has to first investigate the behaviour of the local conditions defining the Selmer groups with respect to the congruences, in other words to ask whether $H_{f}^{1}(K, T)$ varies $p$-adically continuously with $T$ (where now $K$ is a $p$-adic field and $T$ a $Z_{p}$-representation of $G_{K}$ as at the beginning of this introduction). This is the main task of this article.

We will now be more precise and consider two crystalline $Z_{p}$-representations of $G_{K}, T_{1}$ and $T_{2}$ which are isomorphic modulo $p^{n}$ (we say that $T_{1}$ and $T_{2}$ are congruent modulo $\left.p^{n}\right)$. Consider a class $\xi \in H^{1}\left(K, T_{1} / p^{n} T_{1}\right)$ which is the reduction modulo $p^{n}$ of a class coming from $H_{f}^{1}\left(K, T_{1}\right)$. We would like to know if the image of $\xi$ via the isomorphism between $H^{1}\left(K, T_{1} / p^{n} T_{1}\right)$ and $H^{1}\left(K, T_{2} / p^{n} T_{2}\right)$ is the reduction of a class in $H_{f}^{1}\left(K, T_{2}\right)$. This could be reformulated as follows: given an isomorphism l: $T_{1} / p^{n} T_{1} \rightarrow T_{2} / p^{n} T_{2}$ of $Z / p^{n} Z\left[G_{K}\right]$-modules, is there an isomorphism $\tilde{l}$ of $\mathrm{Z} / p^{n} \mathrm{Z}$-modules making the following diagram commutative?

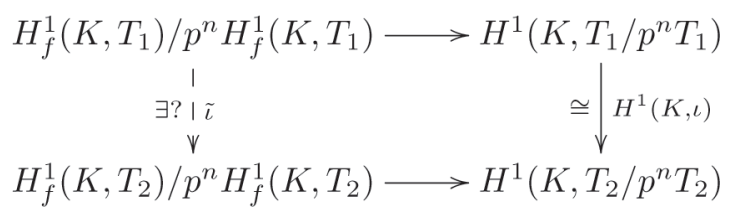

The maps $H_{f}^{1}\left(K, T_{i}\right) / p^{n} H_{f}^{1}\left(K, T_{i}\right) \rightarrow H^{1}\left(K, T_{i} / p^{n} T_{i}\right)$, for $i=1,2$, in the diagram above are induced by the injections $H_{f}^{1}\left(K, T_{i}\right) \hookrightarrow H^{1}\left(K, T_{i}\right)$ and $H^{1}\left(K, T_{i}\right) /$ $p^{n} H^{1}\left(K, T_{i}\right) \hookrightarrow H^{1}\left(K, T_{i} / p^{n} T_{i}\right)$. We recall that they are injective, cf. Lemma 4.1.1, and so this reformulation of the question is only apparently more general. However it makes sense to ask more generally whether $H_{f}^{1}(K,-) / p^{n} H_{f}^{1}(K,-)$ defines a subfunctor of $T / p^{n} T \mapsto H^{1}\left(K, T / p^{n} T\right)$.

It is known that these questions do not have positive answers in general. For example let $T_{1}=Z_{p}(1), T_{2}=Z_{p}(p), K=Q_{p}$ and $p \geq 3$. We have clearly $T_{1} / p T_{1}=\mathbb{F}_{p}(1) \cong T_{2} / p T_{2}$ and Kummer theory implies that $H^{1}\left(\mathrm{Q}_{p}, \mathbb{F}_{p}(1)\right)=$ 
$\mathrm{Q}_{p}^{*} /\left(\mathrm{Q}_{p}^{*}\right)^{p}$, which is a two dimensional $\mathbb{F}_{p}$-vector space. Simple cohomological arguments show that the $\mathbb{F}_{p}$-vector spaces $H_{f}^{1}\left(\mathrm{Q}_{p}, T_{1}\right) / p H_{f}^{1}\left(\mathrm{Q}_{p}, T_{1}\right)$ and $H_{f}^{1}\left(\mathrm{Q}_{p}, T_{2}\right) /$ $p H_{f}^{1}\left(Q_{p}, T_{2}\right)$ have both codimension one in $H^{1}\left(Q_{p}, \mathbb{F}_{p}(1)\right)$. However, one can show that they are orthogonal with respect to cup-product and they correspond to subspaces of $Q_{p}^{*} /\left(Q_{p}^{*}\right)^{p}$ spanned respectively by $p$ and $p+1$. This example shows also that our problem cannot be solved by using only arguments based on the lengthes of the Z/ $/ p^{n} Z_{\text {-modules }} H_{f}^{1}\left(K, T_{i}\right) / p^{n} H_{f}^{1}\left(K, T_{i}\right)$.

Another example, due to R. Greenberg, shows that the equivariance of the morphism $\imath: T_{1} / p^{n} T_{1} \rightarrow T_{2} / p^{n} T_{2}$ with respect to $G_{K}$ is too weak in general. Consider two ordinary elliptic curves $E_{1}$ and $E_{2}$ over $K$, assume that $K$ contains the coordinates of the $p$-torsion points $E_{1}[p]$ and $E_{2}[p]$ and that it has finite residue field. For $i=1,2$, let $T_{i}=T_{p}\left(E_{i}\right)$, where $T_{p}$ denotes the $p$-adic Tate module. A result of Coates and Greenberg (cf. [9]) states that $H_{f}^{1}\left(K, T_{i}\right)$ is equal to the image of $H^{1}\left(K, T\left(\hat{E}_{i}\right)\right) \hookrightarrow H^{1}\left(K, T_{i}\right)$, where $\hat{E}_{i}$ is the formal group of $E_{i}$ (which has height 1 by ordinarity). By the hypothesis on $K$, the reduction $T_{i} / p T_{i}=E_{i}[p]$ is a two dimensional $\mathbb{F}_{p}$-vector space, trivial as $G_{K}$-module, in particular $T_{1}$ and $T_{2}$ are congruent modulo $p$. So any isomorphism $l: T_{1} / p T_{1} \rightarrow T_{2} / p T_{2}$ not sending the image of $T_{p}\left(\hat{E}_{1}\right) / p T_{p}\left(\hat{E}_{1}\right)$ to $T_{p}\left(\hat{E}_{2}\right) / p T_{p}\left(\hat{E}_{2}\right)$ gives a negative answer to our question.

Nevertheless several positive answers are know in the literature, some of them are listed in Remark 1.1.4-(1) : the goal of this article is to generalize them as detailed above.

\section{1 - The main result}

\section{$1.1 .1-$}

We use the notations of the previous section and denote by $K_{0}$ the maximal absolutely unramified subextension of $K$, by $e:=\left[K: K_{0}\right]$ and by $G_{K_{0}}$ the absolute Galois group of $K_{0}$.

Let $n \geq 1$ be an integer, $\mathcal{W} \subset Z_{\text {a subset }}$ and $Z$ a $Z / p^{n} Z_{\text {-module of finite type }}$ endowed with a continuous action of $G_{K}$. We say that $Z$ is crystalline with HodgeTate weights in $\mathcal{W}$ if there exists an exact sequence of $Z_{p}\left[G_{K}\right]$-modules

$$
0 \longrightarrow T^{\prime} \longrightarrow T \longrightarrow Z \longrightarrow 0
$$

where $T$ is a crystalline $Z_{p}$-representation with Hodge-Tate weights in $\mathcal{W}$.

We denote by $\operatorname{Rep}_{Q_{p}}\left(G_{K}\right)_{\text {cris }}^{\mathcal{W}}\left(\operatorname{resp} . \operatorname{Rep}_{Z_{p}}\left(G_{K}\right)_{\text {cris }}^{\mathcal{W}}\right.$, resp. $\left.\operatorname{Rep}_{Z_{/} / p^{n} Z}\left(G_{K}\right)_{\text {cris }}^{\mathcal{W}}\right)$ the category of crystalline $Q_{p}$-representations (resp. Z $Z_{p}$-representations, resp. $Z / p^{n} Z$-representations) with Hodge-Tate weights in $\mathcal{W}$. For semi-stable representations we use analogous notations. For any integers $a \leq b$, we denote by $[a, b]$ the set of integers $i$, such that $a \leq i \leq b$.

Let denote by $\operatorname{Mod}\left(Z / p^{n} Z\right)$ the category of $Z_{/} / p^{n} Z_{4}$-modules. 


\subsection{2 -}

To address the problem of continuity for the Bloch-Kato finite cohomology we proceed as follows. First assume $p \geq 3$. For every integer $0 \leq r \leq p-2$, we construct explicitly a functor

$$
H_{r}^{1}(K,-): \operatorname{Rep}_{Z / p^{n} Z}\left(G_{K_{0}}\right)_{c r y s}^{[0, r]} \rightarrow \operatorname{Mod}\left(Z / p^{n} Z\right),
$$

endowed with a morphism of functors $t_{\mathrm{st}}^{\infty}: H_{r}^{1}(K,-) \rightarrow H^{1}(K,-)$, such that for every crystalline $Z_{p}$-representations $T$ of $G_{K_{0}}$ with Hodge-Tate weights in [0,r], we have the canonical factorisation:

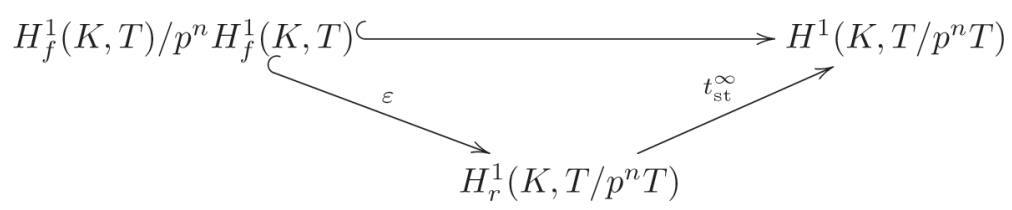

For any crystalline $Z_{p}$-representation $T$ of $G_{K_{0}}$, we denote by $r_{\max }$ or $r_{\max }(T)$ the largest Hodge-Tate weight of $T$.

The following statement which is proved as Theorem 4.1.6 in section $\$ 4.1$ constitutes the main result of this article.

THEOREM. Let $p \geq 3$ be a prime integer and fix an integer $r$ with $0 \leq r \leq p-2$. We denote by $T$ a crystalline $Z_{p}$-representation of $G_{K_{0}}$ with Hodge-Tate weights in $[0, r]$ and assume $e\left(r_{\max }-1\right) \leq p-1$. Then for every integer $n \geq 1, H_{f}^{1}(K, T) /$ $p^{n} H_{f}^{1}(K, T)$ is isomorphic to $H_{r}^{1}\left(K, T / p^{n} T\right)$ via $\varepsilon$.

REMARK 1.1.3.

(1) We point out that if $r_{\max } \leq 1$ the condition on the ramification index $e$ of $K$ is empty.

(2) The hypothesis $e\left(r_{\max }-1\right) \leq p-1$ is necessary, see Proposition 4.2.3.

(3) For every $r$ such that $r_{\max } \leq r \leq p-2$, we have $H_{r}^{1}\left(K, T / p^{n} T\right)=$ $H_{r_{\max }}^{1}\left(K, T / p^{n} T\right)$ canonically. It is a consequence of the theorem but actually it follows already by the construction of $H_{r}^{1}$, see Remark 4.1.3.

In particular if $e(r-1) \leq p-1, H_{r}^{1}(K,-)$ is a subfunctor of $H^{1}(K,-)$; in this case set

$$
H_{f}^{1}(K,-):=H_{r}^{1}(K,-) .
$$

The next statement which is proved as Corollary 4.1.12 in section $\$ 4.1$ states the hypothesis under which the correspondence $T / p^{n} T \mapsto H_{f}^{1}(K, T) / p^{n} H_{f}^{1}(K, T)$ is welldefined and functorial (we have indeed $H_{f}^{1}(K, T) / p^{n} H_{f}^{1}(K, T)=H_{f}^{1}\left(K, T / p^{n} T\right)$ ). 
Corollary. Let $p \geq 3$ and let $T_{1}$ and $T_{2}$ be two crystalline $Z_{p}$-representations of $G_{K_{0}}$ with Hodge-Tate weights in $[0, r] \subseteq[0, p-2]$ and assume $e(r-1) \leq p-1$. Then for every morphism (resp. isomorphism) $\quad l: T_{1} / p^{n} T_{1} \rightarrow T_{2} / p^{n} T_{2}$ of $\mathrm{Z} / p^{n} \mathrm{Z}\left[G_{K_{0}}\right]$-modules, there exists a morphism (resp. isomorphism) i of Z/ $/ p^{n} Z_{-}$ modules making the following diagram commutative.

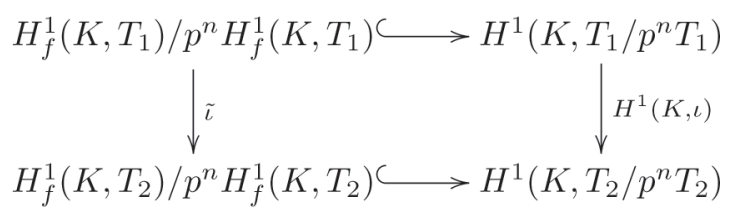

REMARK 1.1.4.

(1) Some cases of Corollary 4.1.12 were already known:

(a) The case where $e=1$ and the representations $T_{1}$ and $T_{2}$ have Hodge-Tate weights in $[0, p-2]$. In this case the result follows from [1, Lemmas 4.4 and 4.5] using Fontaine-Lafaille theory.

(b) In the case where the Hodge-Tate weights of $T_{1}$ and $T_{2}$ are in [0,1], under the hypothesis $\left(T_{1} / p^{n} T_{1}\right)^{G_{K_{0}}}=\left(T_{1} / p^{n} T_{1}\right)^{G_{K}}$ and $p \geq 3$, the result was proved in [13].

(c) The case of representations coming from Barsotti-Tate groups have been treated by Nekovar [18, A.2.6] using flat cohomology; if $p \geq 3$ this implies, by a result of Kisin [16, (2.2.6-7)], the case where representations have Hodge-Tate weights in $[0,1]$.

(2) Under the stronger hypothesis $e r \leq p-2$ we can prove a more general functoriality, see Proposition 4.2.1.

(3) The hypothesis $e(r-1) \leq p-1$ is not necessary in Corollary 4.1.12. For example, assume that $k$ is finite and let $T$ be a crystalline $Z_{p}$-representation of rank one and Hodge-Tate weight $r \geq 2$. We have $H_{f}^{1}(K, T)=H^{1}(K, T)$, with no restrictions on $e$ (cf. [1, Example 3.9]); by Tate duality it follows $H_{f}^{1}(K, T) / p^{n} H_{f}^{1}(K, T)=H^{1}\left(K, T / p^{n}\right)$ and the functoriality is obvious.

(4) If the representations have Hodge Tate weights in $[0, r] \subseteq[0, p-2]$, but not in $[1, r]$, then we do not know if the hypothesis $e(r-1) \leq p-1$ is necessary in Corollary 4.1.12. In particular, we do not know if Corollary 4.1.12 is true even in the following particular case: the representations $T_{1}$ and $T_{2}$ are irreducible of rank 2 with Hodge-Tate weights exactly 0 and $p-2$, with $p \geq 7$ and $e>1$ (or $p=5$ and $e>2$ ).

(5) In Appendix A we deal with the case of characteristic $p=2$ which is essentially independent from the rest of the paper. 


\section{2 - Notation and conventions}

\section{$1.2 .1-$}

We denote by $\mathcal{O}_{\bar{K}}$ (resp. $\mathcal{O}_{K}$ ) the ring of integers of $\bar{K}$ (resp. $K$ ).

For any ring $A$, we denote by $W(A)$ the ring of Witt vectors with coefficients in $A$. When $A=k$, we write simply $W=W(k)$. We recall that we have denoted by $K_{0}$ the field of fractions of $W$ and let $\sigma$ denote the arithmetic Frobenius of $W$ (and $K_{0}$ ), i.e. the isomorphism lifting the $p$-power map on $k$. We set for every $n \geq 0$, $W_{n}=W / p^{n} W$.

We denote by $\mathbb{N}$ the set of integers $i \geq 0$.

\section{Strongly divisible modules}

In the following, except for the appendix, we assume $p>2$.

\section{1 - The ring $S$}

In this section we recall a definition of G. Faltings in [10, §2], see also [5] and [6].

\section{$2.1 .1-$}

Let $\pi$ be a uniformizer of $K$ and denote by $E(u)$ its minimal monic polynomial over $K_{0}$. It is an Eisenstein polynomial with coefficients in $W$ of degree $e$.

We denote by $\mathrm{ev}_{\pi}: W[u] \rightarrow \mathcal{O}_{K}$ the $W$-algebra homomorphism sending $u$ to $\pi$. Its kernel is generated by $E(u)$. We let $S$ denote the $p$-adic completion of the divided power hull of $W[u]$ with respect to the ideal $\operatorname{ker}\left(\mathrm{ev}_{\pi}\right)$, on which we have divided powers compatible with the canonical divided powers of the ideal $p W[u]$. The ring $S$ can be identified to the sub-ring of $\mathrm{Q}_{p} \llbracket u \rrbracket$ of series $\sum_{i \in \mathbb{N}} a_{i} \frac{u^{i}}{q(i) !}$ such that the $a_{i}$ 's belong to $W$, the sequence $\left(a_{i}\right)_{i \geq 0}$ converges to 0 for $i$ going to $+\infty$ and $q(i)$ is the quotient of the Euclidean division of $i$ by $e$.

\section{$2.1 .2-$}

The ring $S$ is endowed with the following extra structures.

(1) A Frobenius homomorphism $\varphi: S \rightarrow S$, which is the unique continuous $\sigma$ semi-linear morphism sending $u$ to $u^{p}$.

(2) A decreasing filtration $\left(\mathrm{Fil}^{i} S\right)_{i \in \mathrm{N}}$ on $S$, defined as follows: for every integer $i \geq 0$, Fil ${ }^{i} S$ is the the $p$-adic closure in $S$ of the ideal generated by $\frac{E(u)^{j}}{j !}$, for all $j \geq i$. We have: for all $0 \leq i \leq p-1, \varphi\left(\mathrm{Fil}^{i} S\right) \subseteq p^{i} S$; for such an integer $i$, put $\varphi_{i}=\left.p^{-i} \varphi\right|_{\mathrm{Fil}^{i} S}$.

Finally, we set $c_{1}=\varphi_{1}(E(u))$ : it is a unit in $S$. 


\subsection{3-}

We denote by $\Omega_{\log }^{1}=\widehat{\Omega}_{S / W}^{1}(\log )\left(\right.$ resp. $\left.\Omega^{1}=\widehat{\Omega}_{S / W}^{1}\right)$ the module of continuous logarithmic differential (resp. regular) 1-forms of $S$ over $W$ and $d: S \rightarrow \Omega^{1} \subset \Omega_{\log }^{1}$ the canonical differential. We have $\Omega^{1}=S d u$ and $\Omega_{\log }^{1}=S u^{-1} d u$.

We denote again $\varphi: \Omega_{\log }^{1} \rightarrow \Omega_{\log }^{1}$ the $\varphi$-semi-linear homomorphism, induced by the Frobenius on $S$ via the universal property of the differentials forms. It maps $u^{-1} d u$ to $p u^{-1} d u$ and we put $\varphi_{1}=p^{-1} \varphi$. The submodule $\Omega^{1}$ is stable under $\varphi$ and $\varphi_{1}$. Clearly we have $d \varphi=p \varphi d$. In the literature, it is also common to introduce the continuous $W$-derivation $N: S \rightarrow S$ sending $u$ to $-u$.

2.2 - Strongly divisible lattices over S (Following [5] and [17])

Let $r$ be an integer such that $0 \leq r \leq p-2$.

$2.2 .1-$

We first define the category ${ }^{\prime} \operatorname{Mod}(S)_{\log }^{r}$. Its objects are 4-uples $\left(M, \mathrm{Fil}^{r} M, \varphi_{r}, \nabla_{M}\right)$, where:

(1) $M$ is an $S$-module;

(2) $\mathrm{Fil}^{r} M$ is a sub-S-module of $M$ containing $\left(\mathrm{Fil}^{r} S\right) M$;

(3) $\varphi_{r}$ is a $\varphi$-semi-linear map $\varphi_{r}: \mathrm{Fil}^{r} M \rightarrow M$, such that for all $s$ in Fil $^{r} S$ and $x$ in $M$ we have $\varphi_{r}(s x)=c_{1}^{-r} \varphi_{r}(s) \varphi_{r}\left(E(u)^{r} x\right)$;

(4) $\nabla_{M}: M \rightarrow M \otimes_{S} \Omega_{\log }^{1}$ is a logarithmic connection satisfying $E(u) \nabla_{M}\left(\mathrm{Fil}^{r} M\right) \subseteq$ $\mathrm{Fil}^{r} M \otimes_{S} \Omega_{\log }^{1}$ such that the following diagram commutes.

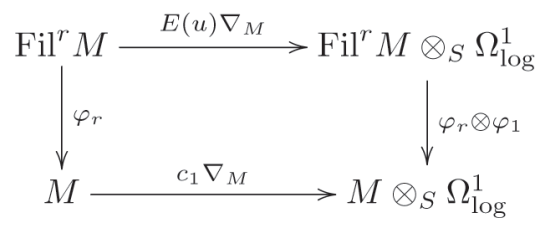

When there is no risk of confusion, we will simply denote $\nabla=\nabla_{M}$.

The morphisms in this category are defined to be the $S$-linear maps preserving the submodule $\mathrm{Fil}^{r}$ and commuting with $\varphi_{r}$ and $\nabla$.

We remark that the integer $r$ is fixed in the definition of the category ${ }^{\prime} \operatorname{Mod}(S)_{\log }^{r}$ and thus the datum of $\mathrm{Fil}^{r} M$ in the 4-uple $\left(M, \mathrm{Fil}^{r} M, \varphi_{r}, \nabla_{M}\right)$ refers to a single submodule of $M$.

If no confusion is arising, we will sometimes denote an object $\left(M, \mathrm{Fil}^{r} M, \varphi_{r}, \nabla\right)$ only by its underlying module $M$.

The category ${ }^{\prime} \operatorname{Mod}(S)_{\log }^{r}$ is $Z_{p}$-linear. A short sequence $0 \rightarrow M^{\prime} \rightarrow M \rightarrow$ $M^{\prime \prime} \rightarrow 0$ of objects in ${ }^{\prime} \operatorname{Mod}(S)_{\log }^{r}$ is said exact, if the induced sequences 
$0 \rightarrow M^{\prime} \rightarrow M \rightarrow M^{\prime \prime} \rightarrow 0$ and $0 \rightarrow \mathrm{Fil}^{r} M^{\prime} \rightarrow \mathrm{Fil}^{r} M \rightarrow \mathrm{Fil}^{r} M^{\prime \prime} \rightarrow 0$ are short exact sequences of $S$-modules.

We denote ${ }^{\prime} \operatorname{Mod}(S)^{r}$ the full subcategory of ${ }^{\prime} \operatorname{Mod}(S)_{\log }^{r}$ of objects whose connection $\nabla$ is regular. In the literature it is common to use the derivation $N_{M}=\nabla_{M}\left(-u \frac{d}{d u}\right)$ instead of the connection $\nabla$ : it is called the monodromy operator and the regularity condition is equivalent to $N(M) \subset u N(M)$.

\subsection{3 - Free objects}

We denote $\operatorname{Mod}(S)^{r}\left(\operatorname{resp} . \operatorname{Mod}(S)_{\log }^{r}\right)$ the full subcategory of ${ }^{\prime} \operatorname{Mod}(S)^{r}(\operatorname{resp}$. $\left.{ }^{\prime} \operatorname{Mod}(S)_{\log }^{r}\right)$ whose objects satisfy:

(1) $M$ is free of finite type as an $S$-module;

(2) the $S$-module $M / \mathrm{Fil}^{r} M$ has no $p$-torsion;

(3) the image of $\varphi_{r}$ generates $M$ as an $S$-module.

We call $\operatorname{Mod}(S)^{r}$ (resp. $\operatorname{Mod}(S)_{\log }^{r}$ ) the category of strongly divisible lattices (resp. logarithmic strongly divisible lattices) over $S$ of weight $r$.

\subsection{4 - Torsion objects}

We denote $\operatorname{ModFI}(S)^{r}$ the full subcategory of ${ }^{\prime} \operatorname{Mod}(S)^{r}$ whose objects satisfy:

(1) $M$ is isomorphic to $\bigoplus_{i \in I} S / p^{n_{i}} S$ as an $S$-module, where $I$ is a finite set and $n_{i}$ are positive integers;

(2) the image of $\varphi_{r}$ generates $M$ over $S$.

For every $n \geq 1$, we denote $\operatorname{ModFI}\left(S / p^{n} S\right)^{r}$ the full subcategory of $\operatorname{ModFI}(S)^{r}$ of objects annihilated by $p^{n}$, as $S$-modules.

\section{3 - Functors towards Galois representations}

\subsection{1 -}

We denote by $R$ the projective limit $\lim \mathcal{O}_{\bar{K}} / p \mathcal{O}_{\bar{K}}$, where the transition maps are given by the absolute Frobenii $x \mapsto x^{p}$. We denote by $A_{\text {cris }}$ the $W(R)$-algebra of crystalline periods defined by Fontaine in [11, 2.2.3]. 
Let $\underline{\pi}=\left(\pi^{(n)}\right)_{n \in \mathrm{N}}$ be a compatible sequence of $p^{n}$-roots of $\pi$, i.e. every $\pi^{(n)}$ belongs to $\bar{K}, \pi^{(0)}=\pi$ and $\pi^{(n+1)^{p}}=\pi^{(n)}$. We denote by $[\underline{\pi}] \in W(R) \subset A_{\text {cris }}$ its Teichmüller representative, and, for any $g$ in $G_{K}$, we put $\varepsilon(g)=g([\underline{\pi}]) /[\underline{\pi}]$ in $A_{\text {cris }}$.

\section{$2.3 .2-$}

We recall the definition of a ring introduced by Kato in [15, §3], and denoted afterwards $\widehat{A}_{\text {st }}$, cf. [2] and [4] for more details.

Let $A_{\text {cris }}\langle x\rangle$ be the divided power hull of the polynomial algebra $A_{\text {cris }}[x]$ compatible with the canonical divided powers of the ideal $p A_{\text {cris }}[x]$; for $j \geq 0$, we denote by $\gamma_{j}(x)$ the $j$-th divided power of $x$. The ring $\widehat{A}_{\text {st }}$ is the $p$-adic completion of $A_{\text {cris }}\langle x\rangle$. It is endowed with the following structures:

(1) A filtration: for any integer $i \geq 0$, $\mathrm{Fil}^{i} \widehat{A}_{\text {st }}=\left\{\sum_{n=0}^{+\infty} a_{n} \gamma_{n}(x) \mid a_{n} \in A_{\text {cris }}, \lim _{n \rightarrow+\infty} a_{n}=0, \forall n \leq i, a_{n} \in \mathrm{Fil}^{i-n} A_{\text {cris }}\right\}$.

We note that for every $n \geq 0$, we have $\mathrm{Fil}^{i} \widehat{A}_{\text {st }} \cap p^{n} \widehat{A}_{\text {st }}=p^{n} \mathrm{Fil}^{i} \widehat{A}_{\text {st }}$.

(2) A Frobenius $\varphi$, extending that of $A_{\text {cris }}$ by mapping $x$ to $(1+x)^{p}-1$.

(3) $\mathrm{A} G_{K}$-action extending the action on $A_{\text {cris }}$, defined as follow: for any $g$ in $G_{K}$, $g(x)=\varepsilon(g) x+\varepsilon(g)-1$.

(4) An $S$-algebra structure given by the monomorphism $S \hookrightarrow \widehat{A}_{\text {st }}, u \mapsto$ $[\tilde{\pi}](1+x)^{-1}$. It identifies $S$ to $\left(\widehat{A}_{\mathrm{st}}\right)^{G_{K}}$ and it is compatible with all the others structures.

(5) A $p$-adically continuous connection $\nabla: \widehat{A}_{\text {st }} \rightarrow \widehat{A}_{\text {st }} \otimes_{S} \Omega_{\text {log }}^{1}$, satisfying $\nabla\left(A_{\text {cris }}\right)=0$ and $\nabla(x)=-(1+x) \otimes u^{-1} d u$.

Remark that for every $0 \leq r \leq p-2$, the datum $\left(\widehat{A}_{\mathrm{st}}, \mathrm{Fil}^{r} \widehat{A}_{\mathrm{st}},\left.p^{-r} \varphi\right|_{\mathrm{Fil}^{r}} \widehat{A}_{\mathrm{st}}, \nabla\right)$ belongs to ${ }^{\prime} \operatorname{Mod}(S)_{\log }^{r}$.

We denote by $T_{\mathrm{st}}^{*}: \operatorname{Mod}(S)_{\log }^{r} \rightarrow \operatorname{Rep}_{Z_{p}}\left(G_{K}\right)_{\mathrm{st}}^{[0, r]}$, the contravariant functor defined as follows:

$$
T_{\mathrm{st}}^{*}(M)=\operatorname{Hom}_{{ }^{\prime} \operatorname{Mod}\left(S_{\text {log }}^{r}\right.}^{r}\left(M, \widehat{A}_{\mathrm{st}}\right),
$$

with the $G_{K}$-action induced by that on $\widehat{A}_{\text {st }}$.

The functor $\mathbb{D}_{\text {free }}^{r}: \operatorname{Rep}_{Z_{p}}\left(G_{K}\right)_{\text {st }}^{[0, r]} \rightarrow \operatorname{Rep}_{Z_{p}}\left(G_{K}\right)_{\text {st }}^{[0, r]}$, sending a $Z_{p}$-representation $T$ to its $r$-twisted $Z_{p}$-linear dual $\operatorname{Hom}_{Z_{p}}\left(T, Z_{p}\right)(r)$, is an anti-equivalence of 
categories because, by definition, the objects in $\operatorname{Rep}_{Z_{p}}\left(G_{K}\right)_{\text {st }}^{[0, r]}$ are free as $Z_{p}$ modules. We denote $T_{\mathrm{st}}: \operatorname{Mod}(S)_{\log }^{r} \rightarrow \operatorname{Rep}_{Z_{p}}\left(G_{K}\right)_{\text {st }}^{[0, r]}$ the covariant functor which is the composition of $T_{\mathrm{st}}^{*}$ with $\mathbb{D}_{\text {free }}^{r}$.

In [5, Conj. 2.2.6-(1)] Breuil conjectured that the functor $T_{\mathrm{st}}^{*}$ is an anti-equivalence ${ }^{1}$ ) of categories and this has been proved by T. Liu.

Theorem 2.3.4. (Theorem 2.3.5 in [17]). For $0 \leq r \leq p-2$, the functor $M \mapsto T_{\mathrm{st}}^{*}(M)$ establishes an anti-equivalence between the category of logarithmic strongly divisible lattices of weight $r$ and the category of semi-stable $Z_{p^{-}}$ representations of $G_{K}$ with Hodge-Tate weights in $[0, r]$.

Corollary 2.3.5. If $M$ is in $\operatorname{Mod}(S)^{r}$, then $T_{\mathrm{st}}^{*}(M)$ is crystalline and the restriction $T_{\mathrm{st}}^{*}$ to $\operatorname{Mod}(S)^{r}$ is an equivalence of categories with $\operatorname{Rep}_{Z_{p}}\left(G_{K}\right)_{\text {cris }}^{[0, r]}$.

PRoof. By a result of Breuil [2] the monodromy operator of $D_{\mathrm{st}}\left(T_{\mathrm{st}}^{*}(M) \otimes_{\mathrm{Z}_{p}} \mathrm{Q}_{p}\right)$ is the residue at zero of the connection of $M \otimes_{W} K_{0}$ : the corollary follows.

EXAMPLE 2.3.6. Let $0 \leq r \leq p-2$ be an integer.

(1) The datum $\left(S, \mathrm{Fil}^{r} S, \varphi_{r}, d\right)$ is an object of $\operatorname{Mod}(S)^{r}$ that we denote shortly by $1(r)$.

(2) The datum $(S, S, \varphi, d)$ is an object of $\operatorname{Mod}(S)^{r}$ that we denote by 1 .

We have $T_{\mathrm{st}}(\mathbb{1})=T_{\mathrm{st}}^{*}(\mathbb{1}(r))=Z_{p}$ and $T_{\mathrm{st}}(\mathbb{1}(r))=T_{\mathrm{st}}^{*}(\mathbb{1})=Z_{p}(r)$.

We denote $\widehat{A}_{\text {st }}^{\infty}=\widehat{A}_{\text {st }} \otimes_{W}\left(K_{0} / W\right)$ and endow it with the induced structures. It is an object of ${ }^{\prime} \operatorname{Mod}(S)_{\text {log }}^{r}$ in a straightforward way. We consider the contravariant functor $T_{\mathrm{st}}^{\infty, *}: \operatorname{ModFI}\left(S / p^{n} S\right)^{r} \rightarrow \operatorname{Rep}_{\mathrm{Z} / p^{n} \mathrm{Z}}\left(G_{K}\right)$ defined by

$$
T_{\mathrm{st}}^{\infty, *}(M)=\operatorname{Hom}_{/ \operatorname{Mod}\left(S_{\log }^{r}\right.}\left(M, \widehat{A}_{\mathrm{st}}^{\infty}\right) .
$$

We denote $\mathbb{D}_{\text {tor }}^{r}: \operatorname{Rep}_{Z / p^{n} Z}\left(G_{K}\right) \rightarrow \operatorname{Rep}_{Z / p^{n} Z}\left(G_{K}\right)$ the Pontryagin duality twisted by $r$, which is the functor sending $T$ to $\operatorname{Hom}_{Z_{p}}\left(T, Q_{p} / Z_{p}\right)(r)$. It is an anti-equivalence of categories and we have $\mathbb{D}_{\text {tor }}^{r}(T)=\operatorname{Hom}_{Z / p^{n} Z}\left(T, Z / p^{n} Z\right)(r)$ because $T$ is annihilated by $p^{n}$. We set

$$
T_{\mathrm{st}}^{\infty}(M)=\mathbb{D}_{\mathrm{tor}}^{r}\left(T_{\mathrm{st}}^{\infty, *}(M)\right)
$$

${ }^{1}{ }^{1}$ The notations for $T_{\mathrm{st}}^{*}$ and $T_{\mathrm{st}}$ are exchanged in [17]. 
By $[4,2.3 .1 .1-2.3 .1 .3]$ the functor $T_{\mathrm{st}}^{\infty, *}$ is exact and so the functor $T_{\mathrm{st}}^{\infty}$ is also exact. In general the functor $T_{\mathrm{st}}^{\infty}$ is not full, but if $e r \leq p-2$ it is fully faithful, cf. [8, Théorème 1.0.4].

For every integer $n \geq 1$, we have the following commutative diagram of functors

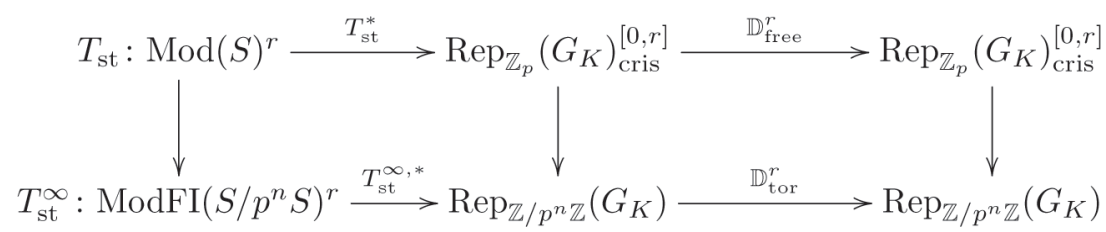

where the vertical functors are reduction modulo $p^{n}$ : indeed by $[7,1.2 .2]$ we have

$$
0 \rightarrow T_{\mathrm{st}}^{*}(M) \stackrel{p^{n}}{\rightarrow} T_{\mathrm{st}}^{*}(M) \rightarrow T_{\mathrm{st}}^{\infty, *}\left(M / p^{n} M\right) \rightarrow 0,
$$

which gives the commutativity of the left square; the commutativity of the right square is obvious because the representations in $\operatorname{Rep}_{Z_{p}}\left(G_{K}\right)_{\text {cris }}^{[0, r]}$ are free $Z_{p}$-modules of finite type.

\section{Extensions of crystalline representations}

\section{1 - The Fontaine-Lafaille theory}

We recall some definitions and results of Fontaine and Lafaille, see [12], [21, §2] and $[1, \S 4]$.

Let $0 \leq r \leq p-2$ be an integer.

\section{$3.1 .1-$} data:

Let $\mathrm{MF}_{W}^{[0, r]}$ be the category whose objects $\left(D, \mathrm{Fil}^{i} D, \varphi_{i}, i \in Z\right)$ are the following

(1) a $W$-module of finite type $D$;

(2) a decreasing filtration $\left(\mathrm{Fil}^{i} D\right)_{i \in \mathrm{Z}}$ by direct summands, satisfying $\mathrm{Fil}^{r+1} D=0$ and $\mathrm{Fil}^{0} D=D$

(3) for all $i$, a $\sigma$-linear map $\varphi_{i}:$ Fil $^{i} D \rightarrow D$, such that $\left.\varphi_{i}\right|_{\mathrm{Fil}^{i+1}}=p \varphi_{i+1}$ and

$$
\sum_{i=0}^{r} \varphi_{i}\left(\mathrm{Fil}^{i} D\right)=D
$$

The morphisms of $\mathrm{MF}_{W}^{[0, r]}$ are $W$-linear maps preserving the filtrations and commuting with the $\varphi_{i}{ }^{\prime}$ s, for all $i$. 


\subsection{2 -}

We say that an object $D$ of $\mathrm{MF}_{W}^{[0, r]}$ is a strongly divisible lattice if it is free as $W$ module. We denote $\mathrm{MF}_{W \text {,free }}^{[0, r]}$ the full subcategory of $\mathrm{MF}_{W}^{[0, r]}$ of strongly divisible lattices. We denote $\mathrm{MF}_{W_{n}}^{[0, r]}$ the full subcategory of $\mathrm{MF}_{W}^{[0, r]}$ whose objects are finite lenght $W_{n}$-modules.

\subsection{3-}

The Fontaine-Lafaille theory gives a commutative diagram of functors

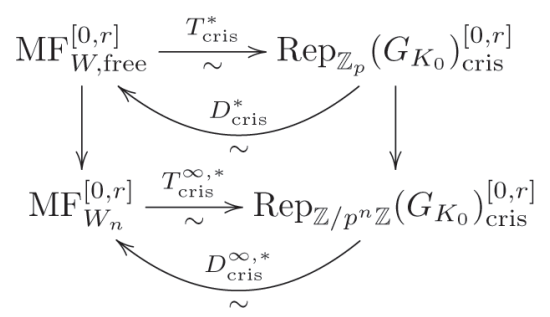

where the vertical functors are reduction modulo $p^{n}$; the functors $T_{\text {cris }}^{*}$ and $T_{\text {cris }}^{\infty, *}$ are (exact) equivalences of categories defined by

$$
T_{\text {cris }}^{*}=\operatorname{Hom}_{W, \text { Fil }, \varphi .}\left(-, A_{\text {cris }}\right) \text { and } T_{\text {cris }}^{\infty, *}=\operatorname{Hom}_{W, \text { Fil }, \varphi .}\left(-, A_{\text {cris }} / p^{n} A_{\text {cris }}\right) ;
$$

and the quasi-inverses of $T_{\text {cris }}^{*}$ and $T_{\text {cris }}^{\infty, *}$ are given respectively by

$$
D_{\text {cris }}^{*}=\operatorname{Hom}_{Z_{p}\left[G_{K_{0}}\right]}\left(-, A_{\text {cris }}\right) \text { and } D_{\text {cris }}^{\infty, *}=\operatorname{Hom}_{Z / p^{n} Z\left[G_{K_{0}}\right]}\left(-, A_{\text {cris }} / p^{n} A_{\text {cris }}\right) \text {. }
$$

We will need also covariant versions of these equivalences, defined by composing with $\mathrm{D}_{\text {free }}^{r}$ or $\mathrm{D}_{\text {tor }}^{r}$ respectively:

$$
\begin{aligned}
& T_{\text {cris }}:=D_{\text {free }}^{r} \circ T_{\text {cris }}^{*}: D \mapsto \operatorname{Fil}^{r}\left(A_{\text {cris }} \otimes_{W} D\right)^{\varphi_{r}=\mathrm{Id}}, \\
& T_{\text {cris }}^{\infty}:=D_{\text {tor }}^{r} \circ T_{\text {cris }}^{\infty, *}: D \mapsto \operatorname{Fil}^{r}\left(A_{\text {cris }} / p^{n} A_{\text {cris }} \otimes_{W_{n}} D\right)^{\varphi_{r}=\mathrm{Id}}, \\
& D_{\text {cris }}:=D_{\text {cris }}^{*} \circ D_{\text {free }}^{r}: T \mapsto\left(A_{\text {cris }} \otimes_{W} T(-r)\right)^{G_{K_{0}}}, \\
& D_{\text {cris }}^{\infty}:=D_{\text {cris }}^{\infty, *} \circ \mathbb{D}_{\text {tor }}^{r}: T \mapsto\left(A_{\text {cris }} / p^{n} A_{\text {cris }} \otimes_{W_{n}} T(-r)\right)^{G_{K_{0}}} .
\end{aligned}
$$

These covariant equivalences are compatible with reduction modulo $p^{n}$ as in the diagram above for contravariant ones. In particular we will need the following: let $T$ be in $\operatorname{Rep}_{Z_{p}}\left(G_{K_{0}}\right)_{\text {cris }}^{[0, r]}$. To the short exact sequence $0 \rightarrow T \rightarrow T \rightarrow T / p^{n} T \rightarrow 0$ we associate the sequence of filtered modules

$$
0 \rightarrow D_{\text {cris }}(T) \stackrel{p^{n}}{\rightarrow} D_{\text {cris }}(T) \rightarrow D_{\text {cris }}^{\infty}\left(T / p^{n} T\right) \rightarrow 0
$$


which is exact in the sense that, for every $i \in \mathbb{Z}$, the induced sequence

$$
0 \rightarrow \mathrm{Fil}^{i} D_{\text {cris }}(T) \stackrel{p^{n}}{\rightarrow} \mathrm{Fil}^{i} D_{\text {cris }}(T) \rightarrow \mathrm{Fil}^{i} D_{\text {cris }}^{\infty}\left(T / p^{n} T\right) \rightarrow 0
$$

is a short exact sequence of $W$-modules, cf. [21, 2.2.3.1].

REMARK 3.1.4. The covariant functors $D_{\text {cris }}$ and $D_{\text {cris }}^{\infty}$ depend on the choice of $r$, unlike their controvariant variants $D_{\text {cris }}^{*}$ and $D_{\text {cris }}^{\infty, *}$. When we need to be precise we shall denote them by $D_{\text {cris }}^{[0, r]}:=D_{\text {cris }}^{*} \circ \mathbb{D}_{\text {free }}^{r}$ and $D_{\text {cris }}^{\infty,[0, r]}:=D_{\text {cris }}^{*} \circ \mathbb{D}_{\text {tor }}^{r}$. For any integers $0 \leq r_{1} \leq r_{2} \leq p-2$, and any representation $T \in \operatorname{Rep}_{Z_{p}}\left(G_{K_{0}}\right)_{\text {crys }}^{\left[0, r_{1}\right]}$, the strongly divisible lattices $D_{\text {cris }}^{\left[0, r_{1}\right]}(T)$ and $D_{\text {cris }}^{\left[0, r_{2}\right]}(T)$ have the same underlying $W$ module but have filtrations (and Frobenii) shifted as follow: for all $i \in Z$,

$$
\operatorname{Fil}^{i}\left(D_{\text {cris }}^{\left[0, r_{2}\right]}(T)\right)=\operatorname{Fil}^{i+\left(r_{1}-r_{2}\right)}\left(D_{\text {cris }}^{\left[0, r_{1}\right]}(T)\right) \quad \text { and } \quad \varphi_{i}^{\left[0, r_{2}\right]}=\varphi_{i+\left(r_{1}-r_{2}\right)}^{\left[0, r_{1}\right]}
$$

In particular the breaks of the filtration of $D_{\text {cris }}^{\left[0, r_{2}\right]}(T)$ are between $r_{2}-r_{1}$ and $r_{2}$. The same formula is true for $D_{\text {cris }}^{\infty,[0, r]}$.

3.2 - Relation with strongly divisible lattices over $S$

$3.2 .1-$

Let $\left(D, \operatorname{Fil}^{i} D, \varphi_{i}, i \in Z\right)$ be an object of $\mathrm{MF}_{W}^{[0, r]}$. We define an object

$$
M(D)=\left(D \otimes_{W} S, \mathrm{Fil}^{r} M(D), \varphi_{r}, \nabla\right)
$$

of ${ }^{\prime} \operatorname{Mod}(S)^{r}$ as follow:

$$
\operatorname{Fil}^{r} M(D)=\sum_{i=0}^{r} \mathrm{Fil}^{i} D \otimes_{W} \mathrm{Fil}^{r-i} S \subset D \otimes_{W} S,
$$

$\varphi_{r}=\sum_{i=0}^{r} \varphi_{i} \otimes_{W} \varphi_{r-i}$ and $\nabla=\mathrm{Id}_{\mathrm{D}} \otimes d$, i.e. $\nabla\left(m \otimes_{W} \alpha\right)=m \otimes_{W} d(\alpha)$. By using the fact that $S$ belongs to ${ }^{\prime} \operatorname{Mod}(S)^{r}$, it is a straightforward exercise to verify that $M(D)$ belongs to ${ }^{\prime} \operatorname{Mod}(S)^{r}$; it is also clear that $D \mapsto M(D)$ is functorial.

By the definitions of the categories $\operatorname{MF}_{W}^{[0, r]}, \operatorname{MF}_{W_{n}}^{[0, r]}, \operatorname{Mod}(S)^{r}$ and $\operatorname{ModFI}\left(S / p^{n} S\right)^{r}$, we can prove that:

- if $D$ is free over $W$, then $M(D)$ belongs to $\operatorname{Mod}(S)^{r}$;

- if $D$ belongs to $\mathrm{MF}_{W_{n}}^{[0, r]}$, then $M(D)$ belongs to $\operatorname{ModFI}\left(S / p^{n} S\right)^{r}$;

- we have $M(D) / p^{n} M(D)=M\left(D / p^{n} D\right)$.

The only non trivial property to check is that $\varphi_{r}\left(\mathrm{Fil}^{r} M(D)\right)$ generates $M(D)$ as an $S$ module. Using (3.2.1.1), it is enough to check that every element of $M(D)=D \otimes_{W} S$ of the form $v \otimes_{W} 1$ is in the image of $\sum_{i=0}^{r} \varphi_{i} \otimes_{W} \varphi_{r-i}$. By hypothesis $v=\sum_{i=0}^{r} \varphi_{i}\left(f_{i}\right)$, for 
some $f_{i} \in \mathrm{Fil}^{i} D$. Put $m_{i}=f_{i} \otimes_{W} E(u)^{r-i} \in \mathrm{Fil}^{i} D \otimes_{W} \operatorname{Fil}^{r-i} S$. Since $\varphi_{r-i}\left(E(u)^{r-i}\right)=$ $c_{1}^{r-i}$ in $S$ we have

$$
\sum_{i=0}^{r} \varphi_{r}\left(m_{i}\right) c_{1}^{-(r-i)}=\sum_{i=0}^{r} \varphi_{i}\left(f_{i}\right) \otimes_{W} \varphi_{r-i}\left(E(u)^{r-i}\right) c_{1}^{-(r-i)}=\sum_{i=0}^{r} \varphi_{i}\left(f_{i}\right) \otimes_{W} 1=v \otimes_{W} 1 .
$$

Proposition 3.2.2. (1) Let $D$ be in $\mathrm{MF}_{W \text {,free }}^{[0, r]}$. We have a natural isomorphism of crystalline Z $Z_{p}$-representations of $G_{K}$

$$
T_{\text {st }}(M(D)) \cong\left(T_{\text {cris }}(D)\right)_{\mid G_{K}} .
$$

(2) Let D be in $\mathrm{MF}_{W_{n}}^{[0, r]}$. We have a natural isomorphism of crystalline Z/ $/ p^{n} \mathrm{Z}_{\text {- }}$ representations of $G_{K}$

$$
T_{\mathrm{st}}^{\infty}(M(D)) \cong\left(T_{\text {cris }}^{\infty}(D)\right)_{\mid G_{K}} .
$$

Proof. The proofs of (1) and (2) are analogue: let us prove (2) and leave (1) to the reader. By composing with the dual functor $\mathbb{D}_{\text {tor }}^{r}=\operatorname{Hom}_{Z_{p}}\left(-, Z / p^{n} Z\right)(r)$ we are reduced to prove the analogous statements on contravariant functors. Since $A_{\text {cris }} / p^{n} A_{\text {cris }}=\left(\widehat{A}_{\text {st }} / p^{n} \widehat{A}_{\text {st }}\right)^{\nabla=0}$, cf. [3, Lemme 3.1.2.3], we have

$$
\begin{aligned}
& T_{\text {cris }}^{\infty, *}(D)=\operatorname{Hom}_{W, \text { Fil }, \varphi \text {. }}\left(D, A_{\text {cris }} / p^{n} A_{\text {cris }}\right) \\
& =\operatorname{Hom}_{W, \mathrm{Fil}, \varphi}\left(D,\left(\widehat{A}_{\mathrm{st}} / p^{n} \widehat{A}_{\mathrm{st}}\right)^{\nabla=0}\right) \\
& =\operatorname{Hom}_{W, \mathrm{Fil}, \varphi, \nabla}\left(D, \widehat{A}_{\mathrm{st}} / p^{n} \widehat{A}_{\mathrm{st}}\right) \\
& =\operatorname{Hom}_{S, \mathrm{Fil}, \varphi, \nabla}\left(D \otimes_{W} S, \widehat{A}_{\mathrm{st}} / p^{n} \widehat{A}_{\mathrm{st}}\right) \\
& \subseteq \operatorname{Hom}_{{ }_{M} \operatorname{Mod}(S)_{\text {log }}^{r}}\left(D \otimes_{W} S, \widehat{A}_{\mathrm{st}} / p^{n} \widehat{A}_{\mathrm{st}}\right)=T_{\mathrm{st}}^{*}(M(D)) \text {. }
\end{aligned}
$$

Let us prove that the inclusion in the last line is an equality. Let $f: D \otimes_{W} S \rightarrow$ $\widehat{A}_{\mathrm{st}} / p^{n} \widehat{A}_{\mathrm{st}}$ be in $T_{\mathrm{st}}^{*}(M(D))$. A priori we have $f\left(\mathrm{Fil}^{i} M(D)\right) \subseteq \mathrm{Fil}^{i}\left(\widehat{A}_{\mathrm{st}} / p^{n} \widehat{A}_{\mathrm{st}}\right)$ and $f \varphi_{i}=\varphi_{i} f$ only for the last step of the filtration: $i=r$; let us prove this is true for any $i$. Let $d$ be in Fil ${ }^{i} D$, for $i \in[0, r]$. For every $\alpha \in \operatorname{Fil}^{r-i} S$ and $d \in D$, we have $\alpha f(d \otimes 1)=f(d \otimes \alpha) \in \mathrm{Fil}^{r}\left(\widehat{A}_{\mathrm{st}} / p^{n} \widehat{A}_{\mathrm{st}}\right)=\mathrm{Fil}^{r} \widehat{A}_{\text {st }} / p^{n} \mathrm{Fil}^{r} \widehat{A}_{\text {st }}$. In particular for $\alpha=E(u)^{r-i}$, we get

$$
E\left([\underline{\pi}](1+x)^{-1}\right)^{r-i} f(d \otimes 1) \in \mathrm{Fil}^{r} \widehat{A}_{\text {st }} / p^{n} \mathrm{Fil}^{r} \widehat{A}_{\mathrm{st}} .
$$

We can re-write it as $E([\underline{\pi}])^{r-i} f(d \otimes 1)+x \beta$, with $\beta \in \widehat{A}_{\text {st. }}$. Since $f(d \otimes 1)$ belongs to $A_{\text {cris }} / p^{n} A_{\text {cris }}$, we get $E([\pi])^{r-i} f(d \otimes 1) \in \mathrm{Fil}^{r}\left(A_{\text {cris }} / p^{n} A_{\text {cris }}\right)$. Considering that $E([\pi])$ is a generator of $\operatorname{ker}\left(\theta: W(R) \rightarrow \mathcal{O}_{\mathbb{C}_{p}}\right)$, we conclude that $f(d \otimes 1)$ belongs to Fil ${ }^{i}\left(A_{\text {cris }} / p^{n} A_{\text {cris }}\right)$.

Let us check the commutation of $f$ with $\varphi_{i}$, for $i \in[0, r]$. For every $\alpha \in$ Fil $^{r-i} S$ and $d \in \mathrm{Fil}^{i} D$, we have

$$
\begin{aligned}
\varphi_{r-i}(\alpha) f\left(\varphi_{i}(d) \otimes 1\right)=f\left(\varphi_{i}(d) \otimes \varphi_{r-i}(\alpha)\right)=f\left(\varphi_{r}(d \otimes \alpha)\right)=\varphi_{r}(f(d \otimes \alpha)) \\
=\varphi_{r}(\alpha f(d \otimes 1))=\varphi(\alpha) \varphi_{r}(f(d \otimes 1))=\varphi_{r-i}(\alpha) \varphi_{i}(f(d \otimes 1)) .
\end{aligned}
$$


By taking $\alpha=E(u)^{r-i}$, we get $\varphi_{r-i}(\alpha)=c_{1}^{r-i}$, which is invertible; thus we have, for all $i \in[0, r]$ and $d \in \operatorname{Fil}^{i} D, f\left(\varphi_{i}(d \otimes 1)\right)=\varphi_{i}(f(d \otimes 1))$. This finishes the proof.

\section{$3.3-$ A double complex computing extensions}

The goal of this subsection is to compute the groups of extensions of strongly divisible modules over $S$ by using explicit complexes.

For $\left(M, \mathrm{Fil}^{r} M, \varphi_{r}, \nabla\right)$ in ${ }^{\prime} \operatorname{Mod}(S)^{r}$ and $0 \leq r \leq p-2$ we set $\operatorname{Fil}^{r-1} M=$ $\left\{m \in M \mid E(u) m \in \mathrm{Fil}^{r} M\right\}$ and define $\varphi_{r-1}: \mathrm{Fil}^{r-1} M \rightarrow M$ by putting, for $m \in \mathrm{Fil}^{r-1} M$,

$$
\varphi_{r-1}(m)=c_{1}^{-1} \varphi_{r}(E(u) m),
$$

where $c_{1}=\varphi_{1}(E(u))$, cf. (2.1.2). Analogously, we define $\varphi: M \rightarrow M$ by $\varphi(m)=$ $c_{1}^{-r} \varphi_{r}\left(E(u)^{r} m\right)$.

We will need later the following.

Lemma 3.3.2. (1) Let $f \in S$ and $j \geq 1$ be an integer. If $E(u) f \in \mathrm{Fil}^{j} S$ then $f \in \mathrm{Fil}^{j-1} S$.

(2) Let $f \in S / p^{n} S$ and $1 \leq j \leq p-1$ be an integer. If $E(u) f \in \mathrm{Fil}^{j}\left(S / p^{n} S\right)$, then $f \in \mathrm{Fil}^{j-1}\left(S / p^{n} S\right)$.

(3) Let $D$ be either in $\mathrm{MF}_{W \text {,free }}^{[0, r]}$ or in $\mathrm{MF}_{W_{n}}^{[0, r]}$. Set $M=M(D)$. If $r=0$ we have $\mathrm{Fil}^{-1} M=M$ and $\varphi_{-1}=p \varphi$; if $r \geq 1$ we have

$$
\mathrm{Fil}^{r-1} M=\sum_{i=0}^{r-1} \mathrm{Fil}^{i} D \otimes_{W} \mathrm{Fil}^{r-1-i} S \subset D \otimes_{W} S
$$

and $\varphi_{r-1}=\sum_{i=0}^{r-1} \varphi_{i}{ }^{D} \otimes \varphi_{r-1-i}: \mathrm{Fil}^{r-1} M \rightarrow M$.

(4) Let $0 \rightarrow D_{1} \rightarrow D_{2} \rightarrow D_{3} \rightarrow 0$ be a short exact sequence in $\mathrm{MF}_{W}^{[0, r]}$. Then the sequence $0 \rightarrow \mathrm{Fil}^{r-1} M\left(D_{1}\right) \rightarrow \mathrm{Fil}^{r-1} M\left(D_{2}\right) \rightarrow \mathrm{Fil}^{r-1} M\left(D_{3}\right) \rightarrow 0$ induced by functoriality is exact.

Proof. Let us prove (1) and (2). Every element $f \in S$ (resp. $S / p^{n}$ ) can be written as $f=\sum_{i \geq 0} a_{i}(u) E(u)^{[i]}$, where $E(u)^{[i]}$ denotes the divided power of $E(u)$ (i.e. $\left.i ! E(u)^{[i]}=E(u)^{i}\right)$ and $a_{i}(u)$ are polynomials in $W[u]$ of degree smaller than $e$, converging to zero (for $i$ going to infinity). We have $E(u) f=\sum_{i \geq 0} a_{i}(u)(i+1) E(u)^{[i+1]}$. If $E(u) f$ belongs to $\mathrm{Fil}^{j} S\left(\operatorname{resp} . \mathrm{Fil}^{j}\left(S / p^{n} S\right)\right)$ then $a_{i}(u)(i+1)=0$ for $i \leq j-1$, which 
implies $a_{i}(u)=0$ (when $f \in S / p^{n} S, i+1 \leq j \leq p-1$ by hypothesis, so that $i+1$ is invertible).

Let us prove (3). The statement for $r=0$ is clear so let us assume $r \geq 1$. Let assume first $D$ in $\mathrm{MF}_{W \text {.free }}^{[0, r]}$. We recall that $D$ is filtered free, and let $\left(e_{i}\right)_{1 \leq i \leq d}$ be a base adapted to the filtration; $i . e$. for every $i \in[0, r],\left(e_{j}\right)_{1 \leq j \leq \mathrm{rk} \mathrm{Fil}}{ }^{i} D$ is a base of Fil ${ }^{i} D$ (cf. [21, 2.2.2]). Put

$$
{ }^{*} \mathrm{Fil}^{r-1} M=\sum_{i=0}^{r-1} \mathrm{Fil}^{i} D \otimes_{W} \mathrm{Fil}^{r-1-i} S \subseteq D \otimes_{W} S .
$$

The inclusion ${ }^{*} \mathrm{Fil}^{r-1} M \subseteq \mathrm{Fil}^{r-1} M$ is obvious so let us prove the converse. Let $x \in \mathrm{Fil}^{r-1} M$, we may assume $x=e_{s} \otimes f$, for some $f \in S$ and $1 \leq s \leq d$. By hypothesis we have $E(u) x=e_{s} \otimes E(u) f \in \operatorname{Fil}^{r}(M)=\sum_{i=0}^{r} \operatorname{Fil}^{i} D \otimes_{W} \mathrm{Fil}^{r-i} S \subseteq D \otimes_{W} S$. We can write

$$
\begin{aligned}
& E(u) x=e_{s} \otimes_{W} E(u) f=\sum_{i=0}^{r} m_{i} \otimes_{W} g_{i} \quad\left(m_{i} \in \mathrm{Fil}^{i} D, g_{i} \in \mathrm{Fil}^{r-i} S\right) \\
& =\sum_{i=0}^{r} \sum_{j=1}^{\mathrm{rkFil} D}\left(e_{j} \otimes_{W} h_{j, i}\right) \quad\left(h_{j, i} \in \mathrm{Fil}^{r-i} S\right) \\
& =\sum_{j=1}^{d} e_{j} \quad \otimes_{W} \sum_{\substack{0 \leq i \leq r \\
\text { rk k } \mathrm{II}^{i} D \geq j}} h_{j, i} .
\end{aligned}
$$

Therefore $E(u) f=\sum_{i} h_{s, i}$, where the sum is taken over all $i \in[0, r]$ such that $\operatorname{rkFil}{ }^{i} D \geq s$. If we denote by $m$ the maximum of the integers $i$ such that $\operatorname{rkFil}^{i} D \geq s$ (or equivalently $e_{s} \in \mathrm{Fil}^{i} D$ ), then $E(u) f$ belongs to $\mathrm{Fil}^{r-m} S$. By (1) we get $f \in \mathrm{Fil}^{r-m-1} S$ and $x=e_{s} \otimes f \in \mathrm{Fil}^{m} D \otimes \mathrm{Fil}^{r-m-1} S \subseteq{ }^{*} \mathrm{Fil}^{r-1} M$.

To finish the proof of (3) for $D \in \mathrm{MF}_{W}^{[0, r]}$,ree , let us compute the Frobenius:

$$
\begin{aligned}
& \varphi_{r-1}(x)=c_{1}^{-1} \varphi_{r}(E(u) x)=c_{1}^{-1} \varphi_{r}\left(e_{s} \otimes E(u) f\right) \\
& c_{1}^{-1} \varphi_{m}\left(e_{l}\right) \otimes \varphi_{r-m}(E(u) f)=\varphi_{m}\left(e_{s}\right) \otimes \varphi_{r-1-m}(f) .
\end{aligned}
$$

The proof of (3) for $D \in \mathrm{MF}_{W_{n}}^{[0, r]}$ is similar because $D$ admits a lifting $\tilde{D} \in \mathrm{MF}_{W \text {.free }}^{[0, r]}$ : so $M=M(D)=\tilde{D} \otimes W S / p^{n} S$ and we use (2) instead of (1). Finally (4) follows from (3) and the fact that for all $i$,

$$
0 \rightarrow \mathrm{Fil}^{i} D_{1} \rightarrow \mathrm{Fil}^{i} D_{2} \rightarrow \mathrm{Fil}^{i} D_{3} \rightarrow 0
$$

is exact (cf. [21, 2.2.3.1]).

Definition 3.3.3. For $\left(M, \operatorname{Fil}^{r} M, \varphi_{r}, \nabla\right)$ in $^{\prime} \operatorname{Mod}(S)^{r}$, we denote $\operatorname{Fil}^{r} C^{\bullet \bullet \bullet}(M)$ the double complex of $Z_{p}$-modules 
Fil $^{r} C^{\bullet \bullet \bullet}(M)$ :

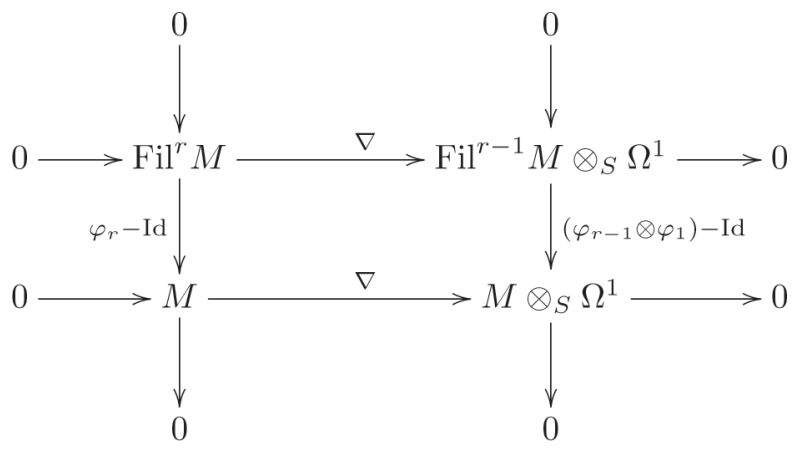

where the module $\mathrm{Fil}^{r} M$ is the $(0,0)$-entry of the complex, and the square is commutative by (2.2.1)-(4) and the definition of $\varphi_{r-1}$, cf. (3.3.1). The simple complex attached to $\mathrm{Fil}^{r} \mathrm{C}^{\bullet \bullet}(M)$ is

$\mathrm{Fil}^{r} C^{\bullet}(M): \quad 0 \rightarrow \mathrm{Fil}^{r} M \stackrel{\nu^{0}}{\rightarrow} M \oplus\left(\mathrm{Fil}^{r-1} M \otimes_{S} \Omega^{1} \stackrel{\gamma^{1}}{\rightarrow} M \otimes_{S} \Omega^{1} \rightarrow 0\right.$, where $\gamma^{0}(m)=\left(\varphi_{r}(m)-m, \nabla_{M}(m)\right)$ and for $a \in M, \omega \in \mathrm{Fil}^{r-1} M \otimes_{S} \Omega^{1}$,

$$
\gamma^{1}(a, \omega)=\nabla_{M}(a)+\omega-\varphi_{r-1} \otimes \varphi_{1}(\omega) .
$$

By construction $H^{i}\left(\operatorname{Fil}^{r} C^{\bullet}(M)\right)=0$ for $i \neq 0,1,2$ and

$$
H^{0}\left(\operatorname{Fil}^{r} C^{\bullet}(M)\right) \cong \operatorname{Hom}_{/ M o d}(S)^{r}(1, M) .
$$

Proposition 3.3.4. We have the following functorial isomorphisms:

(1) if $M$ is in $\operatorname{ModFI}\left(S / p^{n} S\right)^{r}$, then

$$
H^{1}\left(\operatorname{Fil}^{r} C^{\bullet}(M)\right) \cong \operatorname{Ext}_{\operatorname{ModFI}\left(S / p^{n} S\right)^{r}}^{1}\left(1 / p^{n} 1, M\right) ;
$$

(2) if $M$ is in $\operatorname{Mod}(S)^{r}$, then $H^{1}\left(\operatorname{Fil}^{r} C^{\bullet}(M)\right) \cong \operatorname{Ext}_{M \operatorname{Mod}(S)^{r}}^{1}(1, M)$;

where $\mathrm{Ext}^{1}$ denotes the group of Yoneda extensions.

Proof. The proofs of (1) and (2) are analogous. We will prove them by computing explicitly the group of Yoneda extensions and showing that it coincides with $H^{1}\left(\operatorname{Fil}^{r} C^{\bullet}(M)\right)$. An alternative approach would have been to work in the bigger category ${ }^{\prime} \operatorname{Mod}(S)^{r}$ and prove that $H^{1}\left(\mathrm{Fil}^{r} C^{\bullet}(-)\right)$ is an effaçable functor. The problem is that the category ${ }^{\prime} \operatorname{Mod}(S)^{r}$ is not abelian in general and we do not know if there are enough injectives.

The proof takes the rest of this section. Let us start by fixing a convention in order to treat cases (1) and (2) at the same time: for $n \in \mathbb{N} \cup\{\infty\}$, we set

$$
\begin{aligned}
\mathbb{1}_{n} & =\left\{\begin{array}{ll}
1 / p^{n} 1 & \text { if } n \in \mathbb{N} ; \\
1 & \text { if } n=\infty ;
\end{array} \quad S_{n}= \begin{cases}S / p^{n} S & \text { if } n \in \mathbb{N} ; \\
S & \text { if } n=\infty ;\end{cases} \right. \\
\operatorname{Mod}_{n} & = \begin{cases}\operatorname{ModFI}\left(S / p^{n} S\right)^{r} & \text { if } n \in \mathbb{N} ; \\
\operatorname{Mod}(S)^{r} & \text { if } n=\infty .\end{cases}
\end{aligned}
$$


Let $M$ be in $\operatorname{Mod}_{n}$, for some $n \in \mathbb{N} \cup\{\infty\}$, we denote respectively by $\varphi_{r}^{M}$ and $\nabla_{M}$ the Frobenius and the connection of $M$.

Let $[X]$ be a class in $\operatorname{Ext}_{\operatorname{Mod}_{n}}^{1}\left(\mathbb{1}_{n}, M\right)$, represented by a couple of extensions

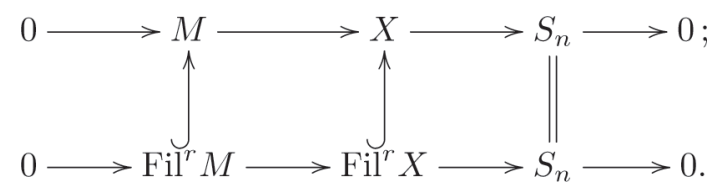

These extensions clearly split as extensions of $S_{n}$-modules (indeed if $n \in \mathbb{N}$, $p^{n} X=0$ by hypothesis); therefore we can write

$$
X=\left(M \oplus S_{n}, \mathrm{Fil}^{r} M \oplus S_{n}, \varphi_{r}^{X}, \nabla_{X}\right) .
$$

Let us describe more precisely the Frobenius $\varphi_{r}^{X}: \mathrm{Fil}^{r} M_{n} \oplus S_{n} \rightarrow M \oplus S_{n}$ and the regular connection $\nabla_{X}: M \oplus S_{n} \rightarrow\left(M \oplus S_{n}\right) \otimes_{S_{n}} S_{n} d u$.

We have $\varphi_{r}^{X}(0,1)=(a, 1)$, for a unique $a \in M$. For all $(m, s) \in \operatorname{Fil}^{r} M \oplus S_{n}$, we get

$$
\begin{aligned}
\varphi_{r}^{X}(m, s)=\left(\varphi_{r}^{M}(m), 0\right)+\varphi(s) \varphi_{r}^{X}(0,1) & =\left(\varphi_{r}^{M}(m), 0\right)+\varphi(s)(a, 1) \\
& =\left(\varphi_{r}^{M}(m)+\varphi(s) a, \varphi(s)\right) .
\end{aligned}
$$

We have $\nabla_{X}(0,1)=(g, 0) \otimes d u$, for a unique $g \in M$. For all $s \in S_{n}$, we get

$$
\begin{aligned}
\nabla_{X}(0, s)=s \nabla_{X}(0,1)+(0,1) \otimes d s & =s(g, 0) \otimes d u+\left(0, \frac{d s}{d u}\right) \otimes d u \\
& =\left(s g, \frac{d s}{d u}\right) \otimes d u ;
\end{aligned}
$$

thus, for all $(m, s) \in M \oplus S_{n}$, we have

$$
\nabla_{X}(m, s)=\left(\nabla_{M}\left(\frac{d}{d u}\right)(m)+s g, \frac{d s}{d u}\right) \otimes d u .
$$

The elements $a, g$ satisfy some compatibility conditions corresponding to those satisfied by $\varphi_{r}^{X}$ and $\nabla_{X}$ (cf. 2.2.1, 2.2.3 and 2.2.4): let us write them explicitly.

Condition 2.2.1-(3): for all $\alpha$ in Fil $^{r} S$ and $x$ in $X$ we have $\varphi_{r}^{X}(\alpha x)=$ $c_{1}^{-r} \varphi_{r}(\alpha) \varphi_{r}^{X}\left(E(u)^{r} x\right)$. For $x=(m, s)$, by using (3.3.4.1), we get

$$
\begin{aligned}
& c_{1}^{-r} \varphi_{r}(\alpha) \varphi_{r}^{X}\left(E(u)^{r} x\right)= \\
& c_{1}^{-r} \varphi_{r}(\alpha) \cdot\left(\varphi_{r}^{M}\left(E(u)^{r} m\right)+\varphi\left(E(u)^{r} s\right) a, \varphi\left(E(u)^{r} s\right)\right)= \\
& \left(c_{1}^{-r} \varphi_{r}(\alpha) \varphi_{r}^{M}\left(E(u)^{r} m\right)+c_{1}^{-r} \varphi_{r}(\alpha) \varphi\left(E(u)^{r} s\right) a, c_{1}^{-r} \varphi_{r}(\alpha) \varphi\left(E(u)^{r} s\right)\right) .
\end{aligned}
$$

By using $\varphi\left(E(u)^{r}\right)=\varphi(E(u))^{r}=\left(p \varphi_{1}(E(u))\right)^{r}=p^{r} c_{1}^{r}$, this is equal to

$$
\begin{aligned}
& \left(\varphi_{r}^{M}(\alpha m)+c_{1}^{-r} \varphi_{r}(\alpha) p^{r} c_{1}^{r} \varphi(s) a, \varphi(\alpha s)\right)= \\
& \left(\varphi_{r}^{M}(\alpha m)+\varphi_{r}(\alpha) p^{r} \varphi(s) a, \varphi(\alpha s)\right)= \\
& \left(\varphi_{r}^{M}(\alpha m)+\varphi(\alpha s) a, \varphi(\alpha s)\right)=\varphi_{r}^{X}(\alpha x),
\end{aligned}
$$

which gives no-condition on $(a, g)$. 
Condition 2.2.1-(4): the regular connection $\nabla_{X}: X \rightarrow X \otimes_{S} \Omega^{1}$ satisfies the Griffith transversality

$$
E(u) \nabla_{X}\left(\mathrm{Fil}^{r} X\right) \subseteq \operatorname{Fil}^{r} X \otimes_{S} \Omega^{1}
$$

and the following diagram commutes.

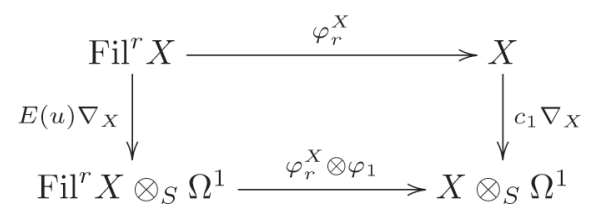

The first condition explicitly gives $E(u) \nabla_{X}(0,1)=(E(u) g, 0) \otimes d u \in \operatorname{Fil}^{r} X \otimes \Omega^{1}$, which is

$$
g \in \mathrm{Fil}^{r-1} M,
$$

cf. 3.3.1 for the definition of $\mathrm{Fil}^{r-1} M$.

For the second one let us compute $\varphi_{r}^{X} \otimes \varphi_{1}\left(E(u) \nabla_{X}(0,1)\right)=\varphi_{r}^{X} \otimes$ $\varphi_{1}((E(u) g, 0) \otimes d u)=\left(\varphi_{r}^{M}(E(u) g), 0\right) \otimes \varphi_{1}(d u) \quad$ and $\quad c_{1} \nabla_{X}\left(\varphi_{r}^{X}(0,1)\right)=c_{1} \nabla_{X}(a, 1)=$ $c_{1}\left(\nabla_{M}(a)+g \otimes d u, 0\right)$. Thus the condition is

$$
\nabla_{M}(a)=c_{1}^{-1} \varphi_{r}^{M}(E(u) g) \otimes \varphi_{1}(d u)-g \otimes d u
$$

or equivalently

$$
\nabla_{M}(a)=\left(\varphi_{r-1}^{M} \otimes \varphi_{1}\right)(g \otimes d u)-g \otimes d u .
$$

The last requirement is that the image of $\varphi_{r}^{X}$ generates $X$, which is always fulfilled because $\varphi_{r}^{M}$ generates $M$ and $\varphi_{r}^{X}(0,1)=(a, 1)$.

We have constructed a surjective map $Z^{1}\left(\mathrm{Fil}^{r} C^{\bullet}(M)\right) \rightarrow \operatorname{Ext}_{\operatorname{Mod}_{n}}^{1}\left(\mathbb{1}_{n}, M\right)$, $(a, g \otimes d u) \mapsto[X]$. The extension $X$ is split if and only if there exists $m \in$ Fil $^{r} M$, such that $m-\varphi_{r}(m)=a$ and $\nabla_{M}(-m)=g \otimes d u$. Indeed, the extension

$$
0 \rightarrow M \rightarrow X \rightarrow \mathbb{1}_{n} \rightarrow 0
$$

is split if and only there is a section $\mathfrak{s}: 1_{n} \rightarrow X$. Giving such a section is equivalent to give $\mathfrak{s}(1)=(m, 1)$, with $m$ satisfying the conditions above. Therefore, the map $(a, g) \mapsto[X]$ induces a bijection of set

$$
H^{1}\left(\operatorname{Fil}^{r} C^{\bullet}(M)\right) \stackrel{\sim}{\rightarrow} \operatorname{Ext}_{\operatorname{Mod}_{n}}^{1}\left(1 / p^{n} 1, M\right) .
$$

To finish we have to prove that this map is a homorphism of groups. Let $\left[X^{(1)}\right]$ and $\left[X^{(2)}\right]$ be extensions associated to couples $\left(a^{(1)}, g^{(1)}\right)$ and $\left(a^{(2)}, g^{(2)}\right)$ respectively. We want to show that the element $\left(a^{(1)}+a^{(2)}, g^{(1)}+g^{(2)}\right)$ corresponds to an extension representing the Baer sum $\left[X^{(1)}\right]+\left[X^{(2)}\right]$. This verification is straightforward: it works essentially because $\varphi^{M}$ and $\nabla_{M}$ are additive. We leave the details to the reader. 
QUESTION 3.3.5. Are there functorial isomorphisms:

(1) for $M$ in $\operatorname{ModFI}\left(S / p^{n} S\right)^{r}, H^{2}\left(\operatorname{Fil}^{r} C^{\bullet}(M)\right) \cong \operatorname{Ext}_{\operatorname{ModFI}\left(S / p^{n} S\right)^{r}}^{2}\left(\mathbb{1} / p^{n} \mathbb{1}, M\right)$ ?

(2) for $M$ in $\operatorname{Mod}(S)^{r}, H^{2}\left(\operatorname{Fil}^{r} C^{\bullet}(M)\right) \cong \operatorname{Ext}_{\operatorname{Mod}(S)^{r}}^{2}(\mathbb{1}, M)$ ?

\section{Proof of the main result}

\section{1 - Construction of $H_{r}^{1}(K,-)$}

Lemma 4.1.1. Let $T$ be a crystalline $Z_{p}$-representation of $G_{K}$. The inclusion $H_{f}^{1}(K, T) \subseteq H^{1}(K, T)$ induces, for every integer $n \geq 1$, an injection

$$
H_{f}^{1}(K, T) / p^{n} H_{f}^{1}(K, T) \hookrightarrow H^{1}\left(K, T / p^{n} T\right) .
$$

Proof. The map $H_{f}^{1}(K, T) / p^{n} H_{f}^{1}(K, T) \rightarrow H^{1}\left(K, T / p^{n} T\right)$ factors through

$$
H_{f}^{1}(K, T) / p^{n} H_{f}^{1}(K, T) \rightarrow H^{1}(K, T) / p^{n} H^{1}(K, T) \rightarrow H^{1}\left(K, T / p^{n} T\right) .
$$

The second morphism is injective since it is part of the long exact sequence associated to $0 \rightarrow T \stackrel{p^{n}}{\rightarrow} T \rightarrow T / p^{n} T \rightarrow 0$.

To show that the first one is injective we have to prove that $H_{f}^{1}(K, T) \cap$ $p^{n} H^{1}(K, T) \subset p^{n} H_{f}^{1}(K, T)$. Let $h$ be a class of a cocycle in $H_{f}^{1}(K, T) \cap p^{n} H^{1}(K, T)$. By definition the image of $h$ in $H^{1}\left(K, T \otimes_{\mathbb{Z}_{p}} \mathrm{~B}_{\text {cris }}\right)$ is trivial and since $H^{1}\left(K, T \otimes_{Z_{p}} \mathrm{~B}_{\text {cris }}\right)$ is a $Q_{p}$-vector space the same is true for the image of $h / p^{n}$. This exactly means that $h$ belongs to $p^{n} H_{f}^{1}(K, T)$.

Definition 4.1.2. For every integers $n \geq 1, i \in\{0,1,2\}$ and $0 \leq r \leq p-2$ we define functors

$$
\begin{aligned}
& H_{r}^{i}(K,-): \operatorname{Rep}_{Z_{p}}\left(G_{K_{0}}\right)_{\text {crys }}^{[0, r]} \rightarrow \operatorname{Mod}\left(Z_{p}\right), \\
& H_{r}^{i}(K,-): \operatorname{Rep}_{Z / p^{n} Z}\left(G_{K_{0}}\right)_{\text {crys }}^{[0, r]} \rightarrow \operatorname{Mod}\left(Z / p^{n} Z\right),
\end{aligned}
$$

by putting, for every $T$ in $\operatorname{Rep}_{Z_{p}}\left(G_{K_{0}}\right)_{\text {crys }}^{[0, r]}$ and $\bar{T}$ in $\operatorname{Rep}_{Z / p^{n} Z}\left(G_{K_{0}}\right)_{\text {crys }}^{[0, r]}$,

$$
H_{r}^{i}(K, T):=H^{i}\left(\operatorname{Fil}^{r} C^{\bullet}\left(M\left(D_{\text {cris }}(T)\right)\right)\right), \quad H_{r}^{i}(K, \bar{T}):=H^{i}\left(\operatorname{Fil}^{r} C^{\bullet}\left(M\left(D_{\text {cris }}^{\infty}(\bar{T})\right)\right)\right),
$$

where $M(-), D_{\text {cris }}(-)$ and $D_{\text {cris }}^{\infty}(-)$ are the functors defined in 3.1.3 and 3.2.1, and $\operatorname{Mod}\left(Z_{p}\right)$ denotes the category of $Z_{p}$-modules.

REMARK 4.1.3. For every $r_{\max } \leq r \leq p-2$ we have $H_{r}^{i}(K, T)=H_{r_{\max }}^{i}(K, T)$ and $H_{r}^{i}(K, \bar{T})=H_{r_{\max }}^{i}(K, \bar{T})$. Indeed by Remark 3.1.4, we have 


$$
\begin{aligned}
\operatorname{Fil}^{r} M\left(D_{\text {cris }}^{[0, r]}(T)\right) & =\operatorname{Fil}^{r}\left(D_{\text {cris }}^{[0, r]}(T) \otimes_{W} S\right) \\
& =\sum_{j=0}^{r} \operatorname{Fil}^{j}\left(D_{\text {cris }}^{[0, r]}(T)\right) \otimes_{W} \operatorname{Fil}^{r-j} S= \\
& =\sum_{j=0}^{r} \operatorname{Fil}^{j+r_{\text {max }}-r}\left(D_{\text {cris }}^{\left[0, r_{\max }\right]}(T)\right) \otimes_{W} \mathrm{Fil}^{r-j} S= \\
& =\sum_{j=r_{\max }-r}^{r_{\max }} \operatorname{Fil}^{j}\left(D_{\text {cris }}^{\left[0, r_{\max }\right]}(T)\right) \otimes_{W} \mathrm{Fil}^{r_{\max }-j} S= \\
& =\sum_{j=0}^{r_{\max }} \mathrm{Fil}^{j}\left(D_{\text {cris }}^{\left[0, r_{\max }\right]}(T)\right) \otimes_{W} \mathrm{Fil}^{r_{\max }-j} S= \\
& =\mathrm{Fil}^{r_{\max }} M\left(D_{\text {cris }}^{\left[0, r_{\max }\right]}(T)\right),
\end{aligned}
$$

and so $H_{r}^{1}(K, T)=H^{i}\left(\operatorname{Fil}^{r} C^{\bullet}\left(M\left(D_{\text {cris }}^{[0, r]}(T)\right)\right)\right)=H^{i}\left(\operatorname{Fil}^{r_{\max }} M\left(D_{\text {cris }}^{\left[0, r_{\max }\right]}(T)\right)\right)=H_{r_{\text {max }}}^{1}(K, T)$. The case of $\bar{T}$ is the same.

Proposition 4.1.4. Let $T$ be in $\operatorname{Rep}_{Z_{p}}\left(G_{K_{0}}\right)_{\text {crys }}^{[0, r]}$, with $0 \leq r \leq p-2$. The short exact sequence

$$
0 \rightarrow T \stackrel{p^{n}}{\longrightarrow} T \rightarrow T / p^{n} T \rightarrow 0
$$

induces two long exact sequences of abelian groups connected as in the commutative diagram below.

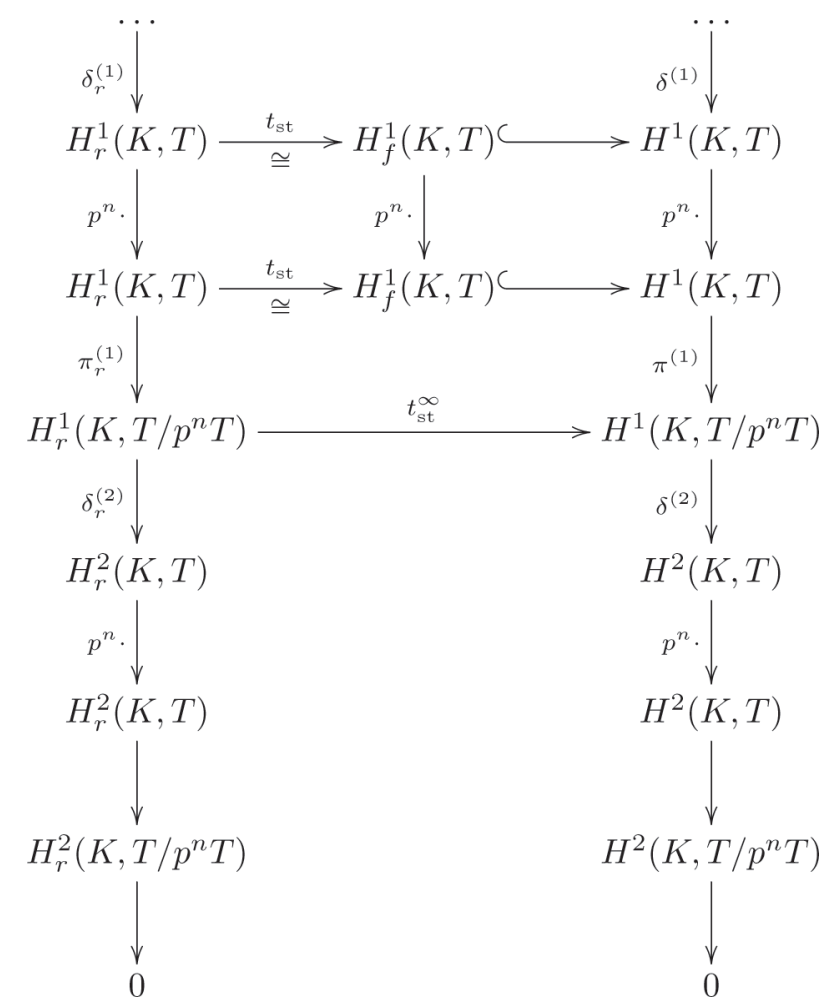


Proof. The right vertical sequence is the long exact sequence of continous Galois cohomology. Set $\bar{T}=T / p^{n} T, D=D_{\text {crys }}(T), \bar{D}=D_{\text {cris }}^{\infty}(\bar{T}), M=M\left(D_{\text {crys }}(T)\right)$ and $\bar{M}=M\left(D_{\text {cris }}^{\infty}(\bar{T})\right)$. We have a short exact sequence $0 \rightarrow D \stackrel{p^{n}}{\rightarrow} D \rightarrow \bar{D} \rightarrow 0$, cf. (3.1.3.1), and by applying $M(-)$ another short exact sequence $0 \rightarrow M \stackrel{p^{n}}{\rightarrow} M \rightarrow \bar{M} \rightarrow 0$, cf. 3.2.1. Therefore, by using Lemma 3.3.2, we have a short exact sequence of complexes

$$
0 \rightarrow \operatorname{Fil}^{r} C^{\bullet}(M) \stackrel{p^{n}}{\rightarrow} \operatorname{Fil}^{r} C^{\bullet}(M) \rightarrow \mathrm{Fil}^{r} C^{\bullet}(\bar{M}) \rightarrow 0 .
$$

The left vertical sequence in (4.1.4.1) is the long exact sequence of cohomology associated to the short exact sequence (4.1.4.2).

To construct $t_{\mathrm{st}}^{\infty}: H_{r}^{1}(K, \bar{T}) \rightarrow H^{1}\left(K, T / p^{n} T\right)$ we proceed as follow.

$$
\begin{aligned}
& H_{r}^{1}(K, \bar{T})=H^{1}\left(\operatorname{Fil}^{r} C^{\bullet}(\bar{M})\right) \\
& \cong \operatorname{Ext}_{\operatorname{ModFI}\left(S / p^{n} S\right)^{r}}^{1}(\overline{\mathbb{1}}, \bar{M}) \\
& \rightarrow \operatorname{Ext}_{\operatorname{Rep}_{Z / p^{n} Z}\left(G_{K}\right)_{\text {crys }}^{[0, r]}}^{1}\left(Z / p^{n} Z, T_{\mathrm{st}}^{\infty}(\bar{M})\right) \quad \text { (by exactness of } T_{\mathrm{st}}^{\infty} \text {, cf. 2.3.7) } \\
& =\operatorname{Ext}_{\operatorname{Rep}_{Z / p^{n} Z}\left(G_{K}\right)_{\text {crys }}^{[0, r]}}^{1}\left(Z / p^{n} Z, \bar{T}_{\mid G_{K}}\right)
\end{aligned}
$$

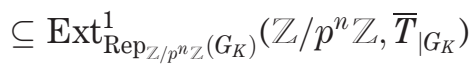

$$
\begin{aligned}
& =H^{1}\left(K, T / p^{n} T\right)
\end{aligned}
$$

To construct the morphism $t_{\text {st }}$ we proceed similarly.

$$
\begin{aligned}
& H_{r}^{1}(K, T)=H^{1}\left(\mathrm{Fil}^{r} C^{\bullet}(M)\right) \\
& =\operatorname{Ext}_{\operatorname{Mod}(S)^{r}}^{1}(1, M) \\
& \stackrel{\sim}{\rightarrow} \operatorname{Ext}_{\operatorname{Rep}_{Z_{p}}\left(G_{K}\right)_{\text {crys }}^{[0, r]}}^{1}\left(Z_{p}, T_{\mathrm{st}}(M)\right) \quad \text { (by Cor. 2.3.5) }
\end{aligned}
$$

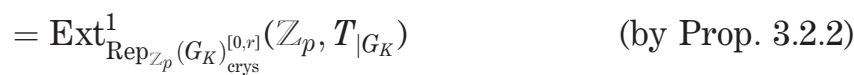

$$
\begin{aligned}
& =H_{f}^{1}(K, T) \quad \text { (by definition) } \\
& \subseteq \operatorname{Ext}_{\operatorname{Rep}_{Z_{p}}\left(G_{K}\right)}^{1}\left(Z_{p}, T_{\mid G_{K}}\right)=H^{1}(K, T) \quad \text { (by definition) }
\end{aligned}
$$

We get a morphism of Z-modules $t_{\mathrm{st}}: H_{r}^{1}(K, T) \rightarrow H^{1}(K, T)$ with image $H_{f}^{1}(K, T)$.

Finally the commutativity for the upper left square in the diagram follows from the additivity of $T_{\text {st }}$, for the upper right square is evident and for the lower square it follows from the the commutativity of the diagram (2.3.7.1).

Corollary 4.1.5. The injection $H_{f}^{1}(K, T) / p^{n} H_{f}^{1}(K, T) \hookrightarrow H^{1}\left(K, T / p^{n} T\right)$, cf. (4.1.1.1), factors canonically through $t_{\mathrm{st}}^{\infty}: H_{r}^{1}\left(K, T / p^{n} T\right) \rightarrow H^{1}\left(K, T / p^{n} T\right)$. We denote by

$$
\varepsilon: H_{f}^{1}(K, T) / p^{n} H_{f}^{1}(K, T) \hookrightarrow H_{r}^{1}\left(K, T / p^{n} T\right)
$$

the monomorphism obtained. 
Proof. The morphism $\varepsilon$ is induced by the composition $\pi_{r}^{(1)} \circ t_{\mathrm{st}}^{-1}$. The factorisation through $t_{\mathrm{st}}^{\infty}$ follows by the commutativity of the diagram (4.1.4.1).

We now state and prove the main result of this article.

THEOREM 4.1.6. Let $p \geq 3$ be a prime integer and we fix an integer $r$ with $0 \leq r \leq p-2$. We consider a crystalline $Z_{p}$-representations of $G_{K_{0}}, T$, with HodgeTate weights in $[0, r]$ and assume $e\left(r_{\max }-1\right) \leq p-1$. Then for every integer $n \geq 1$, $H_{f}^{1}(K, T) / p^{n} H_{f}^{1}(K, T)$ is isomorphic to $H_{r}^{1}\left(K, T / p^{n} T\right)$ via $\varepsilon$.

Proof. Let $T$ be as in Theorem 4.1.6. To show that $\varepsilon: H_{f}^{1}(K, T) / p^{n} H_{f}^{1}(K, T) \rightarrow$ $H_{r}^{1}\left(K, T / p^{n} T\right)$ is an isomorphism we have to prove that $\pi_{r}^{(1)}$ in the diagram (4.1.4.1) is surjective. It is clear that if $H_{r}^{2}(K, T)=0$ (or more generally if $H_{r}^{2}(K, T)$ had not $p$ torsion) then $\pi_{r}^{(1)}$ would be surjective. We will prove below that $H_{r}^{2}(K, T)=0$ if $e=1$ or $r \leq 1$ (cf. Remark 4.1.11) but in general this does not seem to be true. What we do instead is:

(1) we prove that if $H_{r}^{2}\left(K, T / p^{n} T\right)=0$ then $\pi_{r}^{(1)}$ is surjective, see Proposition 4.1.7;

(2) we prove $H_{r}^{2}\left(K, T / p^{n} T\right)=0$ under the hypothesis $e\left(r_{\max }(T)-1\right) \leq p-1$, see Proposition 4.1.10.

Proposition 4.1.7. Let $T$ be in $\operatorname{Rep}_{Z_{p}}\left(G_{K_{0}}\right)_{\text {crys }}^{[0, r]}$ with $r \leq p-2$. If $H_{r}^{2}\left(K, T / p^{n} T\right)=0$ then

$$
\pi_{r}^{(1)}: H_{r}^{1}(K, T) \rightarrow H_{r}^{1}\left(K, T / p^{n} T\right)
$$

is surjective.

Proof. Set $M=M\left(D_{\text {cris }}(T)\right)$ and $\bar{M}=M / p^{n} M$. It is enough to show that the reduction $\bmod p^{n} \operatorname{map} Z^{1}\left(\mathrm{Fil}^{r} C^{\bullet}(M)\right) \rightarrow Z^{1}\left(\mathrm{Fil}^{r} C^{\bullet}(\bar{M})\right)$ on 1-cocycles is surjective. Let $\bar{a} \in \bar{M}$ and $\bar{\gamma} \in \mathrm{Fil}^{r-1} \bar{M} \otimes \Omega^{1}$ be such that

$$
\nabla_{\bar{M}}(\bar{a})=\Psi_{\bar{M}}(\bar{\gamma})
$$

where $\Psi_{\bar{M}}(\bar{\gamma})=\left(\varphi_{r-1}^{\bar{M}} \otimes \varphi_{1}\right)(\bar{\gamma})-\bar{\gamma}$. Let $a_{0} \in M$, (resp. $\left.\gamma_{0} \in \mathrm{Fil}^{r-1} M \otimes \Omega^{1}\right)$ be any lifting of $a$ (resp. $\gamma$ ). We have $\nabla_{M}\left(a_{0}\right)-\Psi_{M}\left(\gamma_{0}\right)=p^{n} \xi_{1}$, for some $\xi_{1} \in M \otimes \Omega^{1}$. Denote by $\bar{\xi}_{1}$ the class of $\xi_{1}$ in $\bar{M} \otimes \Omega^{1}$. Since $H_{r}^{2}\left(K, T / p^{n} T\right)=0$, there exist $\bar{z}_{1} \in \bar{M}$ and $\bar{\omega}_{1} \in \mathrm{Fil}^{r-1} \bar{M} \otimes \Omega^{1}$, such that

$$
\nabla_{\bar{M}}\left(\bar{z}_{1}\right)-\Psi_{\bar{M}}\left(\bar{\omega}_{1}\right)=-\bar{\xi}_{1} .
$$

Take any lifting $z_{1} \in M$ (resp. $\omega_{1} \in \mathrm{Fil}^{r-1} M \otimes \Omega^{1}$ ) of $\bar{z}_{1}$ (resp. $\bar{\omega}_{1}$ ) and set $a_{1}=a_{0}+p^{n} z_{1}$ and $\gamma_{1}=\gamma_{0}+p^{n} \omega_{1}$. We have $\nabla_{M}\left(z_{1}\right)-\Psi_{M}\left(\omega_{1}\right)=-\xi_{1}+p^{n} \xi_{2}$ for some $\xi_{2} \in M \otimes \Omega^{1}$ and

$$
\nabla_{M}\left(a_{1}\right)-\Psi_{M}\left(\gamma_{1}\right)=p^{n} \xi_{1}-p^{n} \xi_{1}+p^{2 n} \xi_{2}=p^{2 n} \xi_{2} .
$$


Since $M$ and $\mathrm{Fil}^{r-1} M \otimes \Omega^{1}$ are $p$-adically complete and separated, it is clear that we can finish by induction.

\section{$4.1 .8-$}

Let us introduce some notations on the ring $S / p S$. We have $E(u) \equiv u^{e} \bmod p$. For any integers $j \geq 0$ and $0 \leq \delta \leq e-1$ we denote by $u^{\langle e j+\delta\rangle}$ the $e$-partial divided power of $u$ (it satisfies $j ! u^{\langle e j+\delta\rangle}=u^{e j+\delta}$ ). We set $\Omega_{S / p S}^{1}=S / p S \otimes_{S} \Omega_{S}^{1}=S / p S d u$. The canonical differential $d: S \rightarrow \Omega_{S}^{1}$ composed with the projection $\Omega_{S}^{1} \rightarrow \Omega_{S / p S}^{1}$ factors through a derivation $S / p S \rightarrow \Omega_{S / p S}^{1}$ that we denote still by $d$. For any $f \in S / p S$ we will write $d f=\frac{d}{d u}(f) \otimes d u$.

LEMMA 4.1.9. Let $f:=\alpha u^{\langle e j+\delta\rangle}$ be in $S / p S$, with $j \geq 0,0 \leq \delta \leq e-1$ and $\alpha \in k^{*}$. Then $f$ is integrable in $S / p S$, i.e. there is $g \in S / p S$ such that $\frac{d}{d u}(g)=f$, if and only if:

- for $\delta \neq e-1, p$ does not divide ej $+\delta+1$;

- for $\delta=e-1, p$ does not divide $e$.

Proof. It is obvious that the conditions are sufficient. Indeed if $\delta \neq e-1$ and $p$ does not divide $e j+\delta+1$, a primitive of $f$ is $\frac{\alpha}{e j+\delta+1} u^{\langle e j+\delta+1\rangle}$; when $\delta=e-1$ and $p \nmid e$, a primitive is $\frac{\alpha}{e} u^{\langle e(j+1)\rangle}$.

The conditions of the lemma are also necessary because if they are not satisfied, we have $\frac{d}{d u}\left(u^{\langle e j+\delta+1\rangle}\right)=0$.

Proposition 4.1.10. Let $T$ be in $\operatorname{Rep}_{Z_{p}}\left(G_{K_{0}}\right)_{\text {crys }}^{[0, r]}$, with $0 \leq r \leq p-2$. For every $n \geq 1$, the $Z / p^{n} Z$-module $H_{r}^{2}\left(K, T / p^{n} T\right)$ is of finite type and if $e\left(r_{\max }(T)-1\right) \leq$ $p-1$, then $H_{r}^{2}\left(K, T / p^{n} T\right)=0$.

Proof. Thanks to Remark 4.1.3 we may assume $r=r_{\max }(T)$. The $n=1$ case implies the case $n \geq 2$ by induction on $n$ and dévissage on the exact sequence

$$
0 \rightarrow p^{n} T / p^{n+1} T \rightarrow T / p^{n+1} T \rightarrow T / p^{n} T \rightarrow 0 .
$$

Let us compute $H_{r}^{2}(K, T / p T)$. Set $\bar{T}=T / p T, D=D_{\text {cris }}(T)$ and $\bar{D}=D_{\text {cris }}^{\infty}(\bar{T})=$ $D / p D$, cf. 3.1.3. By construction the smallest jump in the filtration $\mathrm{Fil}^{i} D$ is $r_{\min }(D)=0$ and the biggest jump is $r_{\max }(D)=r-r_{\min }(T)$, where $r_{\min }(T)$ is the smallest Hodge-Tate weight of $T$. Put $\bar{M}:=M(D) / p M(D) \in \operatorname{ModFI}(S / p S)^{r}$ and $\Psi:=\Psi_{\bar{M}}:=\left(\varphi_{r-1}^{\bar{M}} \otimes \varphi_{1}\right)-\mathrm{Id}$. The idea is to show first that

$$
C:=\operatorname{Coker}\left(\Psi: \operatorname{Fil}^{r-1} \bar{M} \otimes_{S} \Omega^{1} \rightarrow \bar{M} \otimes_{S} \Omega^{1}\right)
$$

is a finite dimensional $\mathrm{F}_{p}$-vector space, which implies of course that $H_{r}^{2}(K, T / p T)$ is 
also finite. Then, we show that, under the hypothesis $e(r-1) \leq p-1$, every element in this cokernel $C$ is in the image of $\nabla_{\bar{M}}$ (see the diagram below), which means $H_{r}^{2}(K, T / p T)=0$.

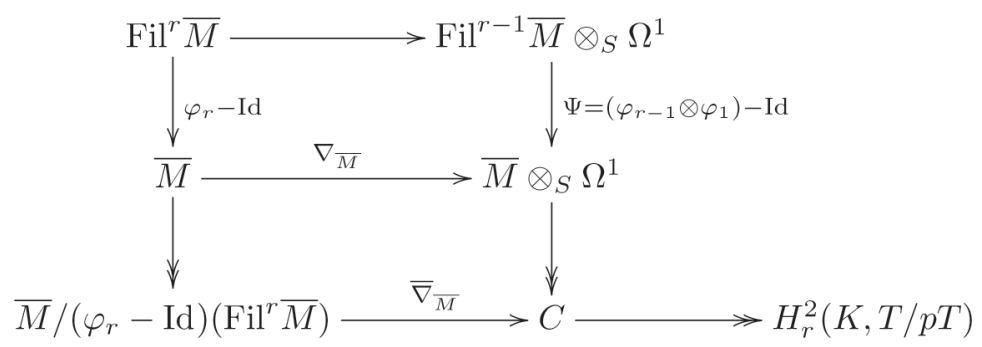

In the particular case $r \leq 1$, the cokernel $C$ is zero. Indeed, in this case, Fil $^{r-1} \bar{M}=\bar{M}$ so that the operator $\varphi_{r-1} \otimes \varphi_{1}: \bar{M} \otimes \Omega^{1} \rightarrow \bar{M} \otimes \Omega^{1}$ can be iterated. The divided Frobenius $\varphi_{1}: \Omega^{1} \rightarrow \Omega^{1}$ is nilpotent:

$$
\varphi_{1}^{m}(d u)=u^{p^{m}-1} d u=\left[\frac{p^{m}-1}{e}\right] ! \frac{u^{p^{m}-1}}{\left[\frac{p^{m}-1}{e}\right] !} d u
$$

therefore $\varphi_{r-1} \otimes \varphi_{1}$ is also nilpotent, $\Psi$ is invertible and $C$ is zero.

For the rest of the proof we suppose $r \geq 2$. Let us prove

$$
\operatorname{dim}_{\mathbb{F}_{p}} C \leq e(r-1) \operatorname{dim}_{F_{p}}(T / p T) .
$$

We write

$$
\bar{M} \otimes_{S} \Omega^{1}=D \otimes_{W} S / p S \otimes_{S} S d u .
$$

Any $\gamma$ in $\bar{M} \otimes_{S} \Omega^{1}$ is a finite sum of elements of the form $v \otimes u^{\langle e j+\delta\rangle} \otimes d u$, where $v \in D, j \geq 0$ and $0 \leq \delta \leq e-1$. We want to find conditions such that $\gamma$ belongs to the image of $\Psi$. It is enough to treat the case $\gamma=v \otimes u^{\langle e j+\delta\rangle} \otimes d u$, and $v \neq 0$. Let $s$ be the weight such that $v \in \mathrm{Fil}^{s} D \backslash \mathrm{Fil}^{s+1} D$; then $\gamma$ belongs to $\mathrm{Fil}^{r-1} \bar{M} \otimes_{S} \Omega^{1}$ if and only if $j \geq r-1-s$.

We compute

$$
\begin{aligned}
\left(\varphi_{r-1} \otimes \varphi_{1}\right)(\gamma) & =\varphi_{s}(v) \otimes \varphi_{r-1-s}\left(u^{\langle e j+\delta\rangle}\right) \otimes u^{p-1} d u \\
& =\varphi_{s}(v) \otimes 1 \otimes \varphi_{r-1-s}\left(\frac{u^{e j+\delta}}{j !}\right) u^{p-1} d u \\
& =\varphi_{s}(v) \otimes 1 \otimes \frac{u^{p(e j+\delta)}}{j ! p^{r-1-s}} u^{p-1} d u \\
& =\varphi_{s}(v) \otimes u^{\langle p(e j+\delta)\rangle} \otimes \frac{\left[\frac{p(e j+\delta)}{e}\right] !}{j ! p^{r-1-s}} u^{p-1} d u .
\end{aligned}
$$


Since $\left[\frac{p(e j+\delta)}{e}\right] \geq p j$, we have

$$
\mathrm{v}_{p}\left(\frac{\left[\frac{p(e j+\delta)}{e}\right] !}{j ! p^{r-1-s}}\right) \geq \mathrm{v}_{p}\left(\frac{p j !}{j ! p^{r-1-s}}\right)=\frac{1}{p-1}\left(p j-\sigma_{p j}-j+\sigma_{j}\right)-(r-1-s),
$$

where, for every $m, \sigma_{m}$ denotes the sum of $p$-adic digits of $m$. Clearly, we have $\sigma_{p j}=\sigma_{j}$ and so

$$
\mathrm{v}_{p}\left(\frac{\left[\frac{p(e j+\delta)}{e}\right] !}{j ! p^{r-1-s}}\right) \geq j-(r-1-s) .
$$

By hypothesis $s \geq r_{\min }(D)=0$; thus if $j \geq r$ then $\varphi_{r-1} \otimes \varphi_{1}(\gamma)=0$, and $\Psi(-\gamma)=\gamma$. If $j=r-1$ then $\left(\varphi_{r-1} \otimes \varphi_{1}\right)(\gamma)$ belongs to $\mathrm{Fil}^{r-1} \bar{M} \otimes_{S} \Omega^{1}$ and the same kind of computation shows that $\left(\varphi_{r-1} \otimes \varphi_{1}\right)^{2}(\gamma)=0$. Thus $\Psi\left(-\gamma-\left(\varphi_{r-1} \otimes \varphi_{1}\right)(\gamma)\right)=\gamma$. In conclusion the element $\gamma=v \otimes u^{\langle e j+\delta\rangle} \otimes d u$ belongs to the image of $\Psi$ for every $j \geq r-1$ and the inequality (4.1.10.1) follows.

To prove $H_{r}^{2}(K, T / p T)=0$ it is enough to show that every $\gamma=v \otimes u^{\langle e j+\delta\rangle} \otimes d u$ in $\bar{M} \otimes \Omega^{1}$, with $v \in D, j \leq(r-2)$ and $0 \leq \delta \leq e-1$, is in the image of $\nabla_{\bar{M}}$. Recall that every $v \in D$ is a horizontal section (by definition of $\nabla_{\bar{M}}$ ) and $e$ is coprime with $p$ (because of $r \geq 2$ and $e(r-1) \leq p-1$ ). By Lemma 4.1.9 we may assume moreover that $0 \leq \delta \leq e-2$ and $e j+\delta+1=p m$ for some integer $m \geq 1$. We have

$$
p m=e j+\delta+1 \leq e(r-2)+\delta+1 \leq(p-1)-e+\delta+1 \leq p-2,
$$

which shows that there are no such elements.

REMARK 4.1.11. It is clear by the proof of Proposition 4.1.10 that if $e=1$ or $r \leq 1$, then $H_{r}^{2}(K, T)=0$. Indeed, if $e=1$, every element in $S$ is integrable, and so $\nabla_{M}$ is surjective. If $r \leq 1$, then $\mathrm{Fil}^{r-1} M=M$, which implies that $\Psi_{M}:=\varphi_{r-1} \otimes \varphi_{1}-$ Id is an isomorphism: the series $\sum_{i=0}^{+\infty}\left(\varphi_{r-1} \otimes \varphi_{1}\right)^{i}$ converges to an inverse of $\Psi_{M}$. This was the idea of the proof in [13]. We do not know if under the hypothesis of Proposition 4.1.10, we have $H_{r}^{2}(K, T)=0$ or not.

End of the proof of Theorem 4.1.6.

Corollary 4.1.12. Let $p \geq 3$ and let $T_{1}$ and $T_{2}$ be two crystalline $Z_{p}$ representations of $G_{K_{0}}$ with Hodge-Tate weights in $[0, r] \subseteq[0, p-2]$ and assume $e(r-1) \leq p-1$. Then for every morphism (resp. isomorphism) $l: T_{1} / p^{n} T_{1} \rightarrow$ $T_{2} / p^{n} T_{2}$ of $Z_{1} / p^{n} Z_{[}\left[G_{K_{0}}\right]$-modules, there exists a morphism (resp. isomorphism) $\tilde{l}$ of $\mathrm{Z}_{1} / p^{n} \mathrm{Z}_{\mathrm{A}}$-modules making the following diagram commutative.

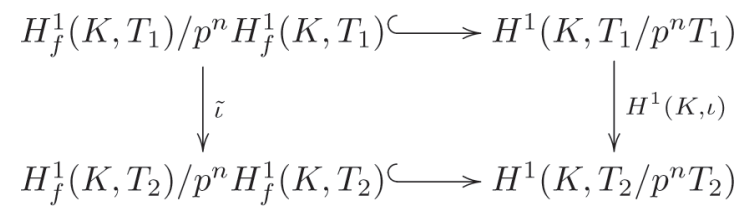


Proof. By the constructions above (cf. (4.1.4.1) and Corollary 4.1.5) we have the following commutative diagram

$$
\begin{gathered}
H_{f}^{1}\left(K, T_{1}\right) / p^{n} H_{f}^{1}\left(K, T_{1}\right) \stackrel{\varepsilon_{1}}{\cong} H_{r}^{1}\left(K, T_{1} / p^{n} T_{1}\right) \stackrel{t_{\mathrm{st}}^{\infty}}{\longrightarrow} H^{1}\left(K, T_{1} / p^{n} T_{1}\right) \\
\qquad H_{r}^{1}(K, \iota) \\
H_{f}^{1}\left(K, T_{2}\right) / p^{n} H_{f}^{1}\left(K, T_{2}\right) \stackrel{\varepsilon_{2}}{\cong} H_{r}^{1}\left(K, T_{2} / p^{n} T_{2}\right) \stackrel{t_{\mathrm{st}}^{\infty}}{\longrightarrow} H^{1}\left(K, T_{2} / p^{n} T_{2}\right)
\end{gathered}
$$

where $\varepsilon_{1}$ and $\varepsilon_{2}$ are isomorphisms by Theorem 4.1.6. Set $\tilde{\imath}=\varepsilon_{2}^{-1} \circ H_{r}^{1}(K, l) \circ \varepsilon_{1}$.

\section{2 - Some complements}

Let us finish this section with a variant of Corollary 4.1.12 and some examples.

Proposition 4.2.1. Let $T_{1}$ and $T_{2}$ be two crystalline $Z_{p}$-representations of $G_{K_{0}}$ with Hodge-Tate weights in $[0, r] \subseteq[0, p-2]$ and assume er $\leq p-2$. Then for every morphism (resp. isomorphism) $\imath: T_{1} / p^{n} T_{1} \rightarrow T_{2} / p^{n} T_{2}$ of Z $/ p^{n} Z\left[G_{K}\right]-$ modules, there exists a morphism (resp. isomorphism) i of Z//pn Z-modules making the following diagram commutative.

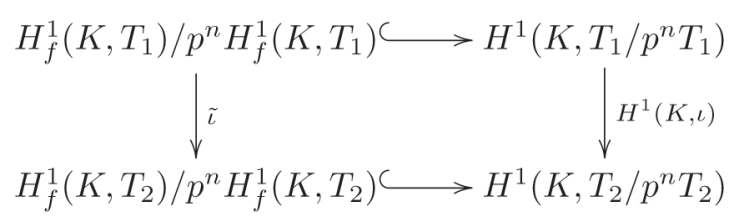

\section{REMARK 4.2.2.}

(1) The hypothesis $e r \leq p-2$ is more restrictive than that of Corollary 4.1.12 (it implies $(e, p)=1$ even for $r=1$ ), but Proposition 4.2.1 gives functoriality for $G_{K}$-morphismes.

(2) The hypothesis $e r \leq p-2$ is necessary. Indeed consider the following counterexample for $e=p-1$ and $r=1$. Take $K=\mathrm{Q}_{p}\left(\mu_{p}\left(\overline{\mathrm{Q}}_{p}\right)\right)$, $T_{1}=Z_{p}(1)$ and $T_{2}=Z_{p}$. Clearly $T_{1}$ and $T_{2}$ are congruent modulo $p$ but $H_{f}^{1}\left(K, T_{1}\right) \otimes z_{p} F_{p}$ and $H_{f}^{1}\left(K, T_{2}\right) \otimes Z_{p} \mathbb{F}_{p}$ do not even have the same dimension over $\mathbb{F}_{p}$.

Proof. The proof is similar but simpler than that of Corollary 4.1.12 so we only sketch it. For $i=1,2$ set $D_{i}=D_{\text {cris }}\left(T_{i}\right)$ and $M_{i}=M\left(D_{i}\right)$; we have $T_{i} / p^{n} T_{i}=$ $T_{\text {st }}^{\infty}\left(M_{i} / p^{n} M_{i}\right)$. Under the hypothesis $e r \leq p-2$, the functor $T_{\text {st }}^{\infty}$ is fully faithful (cf. [8, Theéorème 1.0.4]) therefore the morphism $l$ is induced by a unique 
morphism $\imath^{\prime}: M_{1} / p^{n} M_{1} \rightarrow M_{2} / p^{n} M_{2}$ in $\operatorname{ModFI}\left(S / p^{n} S\right)^{r}$ and we have a commutative diagram

$$
\begin{aligned}
& H_{f}^{1}\left(K, T_{1}\right) / p^{n} H_{f}^{1}\left(K, T_{1}\right) \stackrel{\varepsilon_{1}}{\cong} H^{1}\left(\operatorname{Fil}^{r} C^{\bullet}\left(M_{1} / p^{n} M_{1}\right)\right) \stackrel{t_{\mathrm{st}}^{\infty}}{\longrightarrow} H^{1}\left(K, T_{1} / p^{n} T_{1}\right) \\
& \qquad H^{1}\left(\operatorname{Fil}^{r} C^{\bullet}\left(\iota^{\prime}\right)\right) \\
& H_{f}^{1}\left(K, T_{2}\right) / p^{n} H_{f}^{1}\left(K, T_{2}\right) \stackrel{\varepsilon_{2}}{\cong} H^{1}\left(\operatorname{Fil}^{r} C^{\bullet}\left(M_{2} / p^{n} M_{2}\right)\right) \stackrel{t_{\mathrm{st}}^{\infty}}{\longrightarrow} H^{1}\left(K, T_{2} / p^{n} T_{2}\right)
\end{aligned}
$$

where $\varepsilon_{1}$ and $\varepsilon_{2}$ are isomorphisms by Theorem 4.1.6. Set $\tilde{l}=\varepsilon_{2}^{-1} \circ H^{1}\left(\operatorname{Fil}^{r} C^{\bullet}\left(l^{\prime}\right)\right) \circ \varepsilon_{1}$.

For an abelian group $A$ we denote by $A[n]$ the $n$-torsion subgroup of $A$.

Proposition 4.2.3. Let $2 \leq r \leq p-2$ be an integer and assume $e \geq p$, then:

(1) The groups $H_{r}^{2}\left(K, Z_{p}(r)\right)\left[p^{n}\right]$ and $H_{r}^{2}\left(K, Z / p^{n} Z(r)\right)$ are non-zero.

(2) The monomorphism $\varepsilon: H_{f}^{1}\left(K, Z_{p}(r)\right) / p^{n} H_{f}^{1}\left(K, Z_{p}(r)\right) \hookrightarrow H_{r}^{1}\left(K, T / p^{n} T\right) d e$ fined in Corollary 4.1.5 is not surjective.

Proof. The statement (2) follows from (1) by using (4.1.4.1). Let us prove (1): it is enough to treat the case $n=1$. Set $D=D_{\text {cris }}\left(Z_{p}(r)\right)$. We have $D=$ $D_{\text {cris }}^{*}\left(Z_{p}(-r)(r)\right)=\operatorname{Hom}_{Z_{p}\left[G_{K_{0}}\right]}\left(Z_{p}, A_{\text {cris }}\right)=W, \operatorname{Fil}^{0} D=D, \mathrm{Fil}^{1} D=0$, and $\varphi_{0}^{D}=\sigma$. Hence $M(D)=D \otimes_{W} S=S$, Fil $^{r} M(D)=$ Fil $^{r} S$ as $S$-modules, and $M(D)=\mathbb{1}(r)$, cf. 2.3.6. We have $H_{r}^{1}\left(K, Z_{p}(r)\right)=H^{1}\left(\operatorname{Fil}^{r} C^{\bullet}(1(r))\right)$ and $H_{r}^{1}\left(K, \mathbb{F}_{p}(r)\right)=H^{1}\left(\operatorname{Fil}^{r} C^{\bullet}(\overline{1(r)})\right)$, where $\overline{\mathbb{1}(r)}=\mathbb{1}(r) / p^{n} \mathbb{1}(r)$. Denote by $\xi$ the class of $u^{p-1} \otimes_{S} d u$ in $H^{2}\left(\operatorname{Fil}^{r} C^{\bullet}(1(r))\right)$. We have $d\left(u^{p}\right)=p\left(u^{p-1} \otimes_{S} d u\right)$, so $p \xi=0$. We claim that the class of $u^{p-1} \otimes d u$ in $H^{2}\left(\operatorname{Fil}^{r} C^{\bullet}(\overline{\mathbb{1}(r)})\right)$ is not zero, which implies at the same time $\xi \neq 0, H_{r}^{2}\left(K, \mathbb{F}_{p}(r)\right) \neq 0$ and $H_{r}^{2}\left(K, Z_{p}(r)\right)[p] \neq 0$. Let us prove this claim by contradiction: let $a$ be in $\overline{\mathbb{1}(r)}=S / p S$ and $g \otimes d u$ be in $\mathrm{Fil}^{r-1} \overline{\mathbb{1}(r)} \otimes \Omega^{1}=\mathrm{Fil}^{r-1}(S / p S) \otimes_{S} \Omega^{1}$, such that

$$
\frac{d}{d u}(a) \otimes d u-\left(\varphi_{r-1} \otimes \varphi_{1}\right)(g \otimes d u)+g \otimes d u=u^{p-1} \otimes d u,
$$

or equivalently

$$
\frac{d}{d u}(a)-u^{p-1} \varphi_{r-1}(g)+g=u^{p-1} .
$$

Since $r-1 \geq 1$ and $e \geq p$, by writing $g=\sum_{j \geq r-1} g_{j} u^{\langle e j+\delta\rangle}$ (with $g_{j} \in k$ and $0 \leq \delta \leq e-1)$, and by taking reduction modulo $\mathrm{Fil}^{p}(S / p S)$, we get that there exists $\alpha \in S /\left(p S+\mathrm{Fil}^{p} S\right)=k[u] / u^{p}$, such that

$$
\frac{d}{d u}(\alpha)=u^{p-1}
$$

which is impossible. 
REMARK 4.2.4. Let $2 \leq r \leq p-2$ be an integer, assume $e \geq p$ and that the residue field of $K$ is finite. We have

$$
H_{f}^{1}\left(K, Z_{p}(r)\right) / p H_{f}^{1}\left(K, Z_{p}(r)\right) \stackrel{q}{\hookrightarrow} H_{r}^{1}\left(K, \mathbb{F}_{p}(r)\right) \stackrel{t_{\mathrm{st}}^{\infty}}{\longrightarrow} H^{1}\left(K, \mathbb{F}_{p}(r)\right),
$$

and by [1, Example 3.9, pg. 359] $H_{f}^{1}\left(K, Z_{p}(r)\right)=H^{1}\left(K, Z_{p}(r)\right)$; in particular $t_{\mathrm{st}}^{\infty}$ is surjective but not injective and $H_{f}^{1}\left(K, Z_{p}(r)\right) / p H_{f}^{1}\left(K, Z_{p}(r)\right)$ can be identified to $t_{\mathrm{st}}^{\infty}\left(H_{r}^{1}\left(K, \mathbb{F}_{p}(r)\right)\right)$. We suspect that this phenomenon happens more generally.

Proposition 4.2.5. Let $T$ be a crystalline $Z_{p}$-representation of $G_{K_{0}}$ with Hodge-Tate weights in $[0, r], 0 \leq r \leq p-2$. We have an isomorphism

$$
t_{\mathrm{st}}^{\infty}: H_{r}^{0}\left(K, T / p^{n} T\right) \stackrel{\sim}{\rightarrow}\left(T / p^{n} T\right)^{G_{K}} .
$$

Proof. Set $D=D_{\text {cris }}(T)$ and $M=M(D)$. A direct computation shows

$$
\begin{aligned}
H_{r}^{0}\left(K, T / p^{n} T\right) & =\operatorname{Hom}_{{ }_{M} \operatorname{Mod}(S)^{r}}\left(\mathbb{1} / p^{n} \mathbb{1}, M / p^{n} M\right) \\
& =\operatorname{Fil}^{r}\left(\left(S / p^{n} S\right)^{\nabla=0} \otimes_{W_{n}} D / p^{n} D\right)^{\varphi_{r}=\mathrm{Id}} \\
& \supseteq \operatorname{Fil}^{r}\left(D / p^{n} D\right)^{\varphi_{r}=\mathrm{Id}} \cong\left(T / p^{n} T\right)^{G_{K_{0}}} .
\end{aligned}
$$

The last inclusion is strict in general. Indeed we have the exact sequence

$$
0 \rightarrow H_{r}^{0}(K, T) \stackrel{p^{n}}{\longrightarrow} H_{r}^{0}(K, T) \rightarrow H_{r}^{0}\left(K, T / p^{n} T\right) \stackrel{\delta}{\rightarrow} H_{r}^{1}(K, T) \stackrel{p^{n}}{\longrightarrow} H_{r}^{1}(K, T) \rightarrow
$$

induced by the short exact sequence $0 \rightarrow M \stackrel{p^{n}}{\longrightarrow} M \rightarrow M / p^{n} M \rightarrow 0$. By the theorem of Liu (2.3.4) $T_{\text {st }}$ is an equivalence of categories and therefore we have

$$
H_{r}^{0}(K, T)=\operatorname{Hom}_{{ }_{M} \operatorname{Mod}(S)^{r}}(1, M)=\operatorname{Hom}_{\operatorname{Mod}(S)^{r}}(1, M) \stackrel{\sim}{\rightarrow} T^{G_{K}}
$$

and $H_{r}^{1}(M) \cong H_{f}^{1}(K, T)$, cf. (4.1.4.1). By definition $H_{f}^{1}(K, T)$ contains all the torsion of $H^{1}(K, T)$, so the kernel of $H_{r}^{1}(K, T) \stackrel{p^{n}}{\longrightarrow} H_{r}^{1}(K, T)$ is equal to the $p^{n}$-torsion subgroup $H^{1}(K, T)\left[p^{n}\right]$ of $H^{1}(K, T)$. As in the proof of (4.1.4.1), the exact functors $T_{\mathrm{st}}$ and $T_{\mathrm{st}}^{\infty}$ induce the commutative diagram

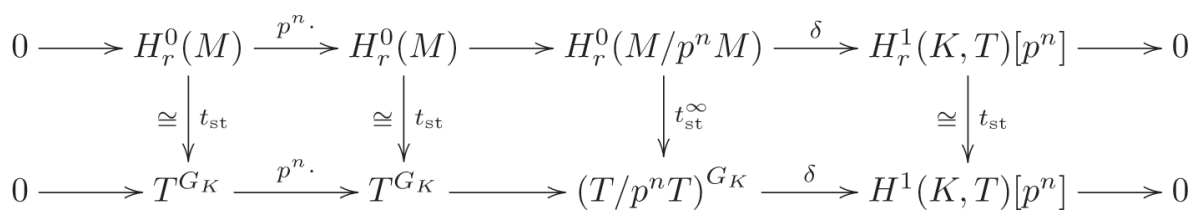

where the rows are exact. This proves the claim.

\section{A. The characteristic two case}

In all this section we assume $p=2$. We will consider only representations with Hodge-Tate weights equal to zero $(r=0)$, otherwise there are counterexamples to the question raised in the introduction (e.g. $Z_{2}$ and $Z_{2}(1)$ are con- 
gruent modulo 2 but $H_{f}^{1}\left(\mathrm{Q}_{2}, \mathrm{Z}_{2}\right) / 2 H_{f}^{1}\left(\mathrm{Q}_{2}, \mathrm{Z}_{2}\right)$ and $H_{f}^{1}\left(\mathrm{Q}_{2}, \mathrm{Z}_{2}(1)\right) / 2 H_{f}^{1}\left(\mathrm{Q}_{2}, \mathrm{Z}_{2}(1)\right)$ have dimension one and two respectively over $\mathbb{F}_{2}$ ). The statements and the proofs given in this appendix work also for a general $p \geq 2$, but the results for $p \geq 3$ are already a consequence of Corollary 4.1.12, thus we state them only for $p=2$.

Theorem A.1. Let $K / Q_{2}$ be a finite extension. Let $T_{1}$ and $T_{2}$ be two crystalline $Z_{2}$-representations of $G_{K}$ with Hodge-Tate weights equal to zero. Let $\imath: T_{1} / 2^{n} T_{1} \rightarrow$ $T_{2} / 2^{n} T_{2}$ be a morphism (resp. isomorphism) of $Z / 2^{n} Z\left[G_{K}\right]$-modules. Then there exists a morphism (resp. isomorphism) $\tilde{i}$ of Z/ $/ 2^{n} \mathrm{Z}$-modules making the following diagram commutative.

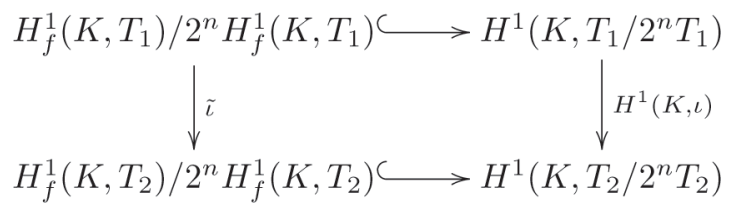

REMARK A.2. Actually from the proof it follows that Theorem 4.2.5 works for any complete discrete valuation field $K$ of mixed characteristic $(0,2)$ whose residue field $k$ has cohomological dimension smaller than one.

Lemma A.3. Let $T$ be crystalline with Hodge-Tate weights equal to zero. We have $H_{f}^{1}(K, T)=H^{1}(k, T)$.

Proof. The Hodge-Tate weights of $T$ are zero so, by a result of Sen [19, Corollary, pg. 114], the inertia subgroup $I_{K}$ of $G_{K}$ acts through a finite quotient; moreover $T$ is crystalline, thus $I_{K}$ acts trivially and $T$ can be considered a $G_{k}$ module.

Let $T^{\prime}$ be an extension representing a class $x$ in $H^{1}(K, T)$. We have $x \in H_{f}^{1}(K, T)$ if and only if $T^{\prime}$ is crystalline with Hodge-Tate weights 0 , if and only if the inertia $I_{K}$ acts trivially on $T^{\prime}$. Thus $H_{f}^{1}(K, T)=\operatorname{ker}\left(H^{1}(K, T) \stackrel{\text { Res }}{\longrightarrow} H^{1}\left(\widehat{K}^{\mathrm{nr}}, T\right)\right)$. By the Inflation-Restriction short exact sequence [20, VII $\S 6$ Prop.4], we have $H_{f}^{1}(K, T)=$ $H^{1}\left(G_{k}, T\right)$.

Proof of Theorem A.1. Apply Lemma (A.3) to the long exact sequence associated to

$$
0 \rightarrow T \stackrel{\cdot 2^{n}}{\longrightarrow} T \rightarrow T / 2^{n} T \rightarrow 0 .
$$

Since $k$ is finite $H^{2}(k, T)=0$ by [20, XIII, $\S 1$, Prop. 2] therefore $H_{f}^{1}(K, T) /$ $2^{n} H_{f}^{1}(K, T)=H^{1}\left(k, T / 2^{n} T\right)$ and the statement follows. 
Acknowledgments. Some part of the work on this article was done while both authors visited the Institute Henri Poincaré (IHP) in Paris during the Galois semester in Winter 2010. We are grateful to the organizers and the IHP for their hospitality. During that semester the second author benefited from a reduction of teaching funded by the project ANR-09-JCJC-0048-01. This article also benefited from several visits of the second author at the University of Padua and Concordia University, we thank these institutions and Bruno Chiarellotto for funding. Part of the work of the first author on this article was funded by the grant PRIN-MIUR 2010-11. "Arithmetic Algebraic Geometry and Number Theory" and an NSERC Discovery grant. We thank Xavier Caruso and Jean-Pierre Wintenberger for useful discussions on matters pertaining to this research.

\section{REFERENCES}

[1] S. Bloch - K. Kato, L-functions and Tamagawa numbers of motives, in: The Grothendieck Festschrift, Vol. I, Progr. Math. 86, Birkhäuser, Boston 1990, P. Cartier, et al., eds., pp. 333-400.

[2] C. BreulL, Représentations p-adiques semi-stables et transversalité de Griffiths, Math. Ann., 307, 1997, pp. 191-224.

[3] C. Breull, Constructions de représentations p-adiques semistables, Ann. sci. E.N.S. $4^{e}$ série, tome 31, n. 3, 1998, pp. 281-327.

[4] C. Breuil, Représentations semi-stables et modules fortement divisibles, Invent. Math., 136, 1999, pp. 89-122.

[5] C. Breuil, Integral p-adic Hodge theory, Advanced Studies in Pure Math., 36, 2002, pp. $51-80$.

[6] C. Breuil - W. Messing, Torsion étale and crystalline cohomologies, in: Cohomologies p-adiques et applications arithmétiques (II), Astérisque, 279, Soc. Math. de France, Paris 2002, pp. 81-124.

[7] X. CaRuso - T. LiU, Quasi-semi-stable representations, Bull. Soc. Math. France, 137, 2009 , n. 2, pp. $185-223$.

[8] X. Caruso, Représentations semi-stables de torsion dans le cas er $<p-1$, J. reine angew. Math., 594, 2006, pp. 35-92.

[9] J. CoAtes - R. GReEnBerG, Kummer theory for abelian varieties over local fields, Invent. Math., 124, 1996, pp. 129-174.

[10] G. FALTings, Integral crystalline cohomology over very ramified valuation rings, J.A.M.S., Volume 12, n. 1, 1999, pp. 117-144.

[11] J.-M. FonTAIne, Le corps de périodes p-adiques, avec une appendice par Pierre Colmez, in: Périodes p-adiques, Astérisque, 223, Soc. Math. de France, Paris 1994, pp. $59-111$.

[12] J.-M. Fontaine - G. Lafaille, Construction de représentations p-adiques, Ann. sci. E.N.S. 4ème série, tome 15, n. 4, 1982, pp. 547-608.

[13] R. Greenberg - A. Iovita - R. Pollack, On I wasawa Invariants of Elliptic Curves at Supersingular Primes, preprint.

[14] R. Greenberg - V. VAtsal, On the Iwasawa invariants of elliptic curves, Invent. Math., 142, 2000, pp. 17-63.

[15] K. Kato, Semi-stable reduction and p-adic étale cohomology, in: Périodes p-adiques, Astérisque, 223, Soc. Math. de France, Paris 1994, pp. 269-293. 
[16] M. Kisin, Crystalline representations and F-crystals, in Algebraic Geometry and Number Theory, Drinfeld 50th Birthday volume, Progress in mathematics, vol. 253, Birkhäuser, Boston, MA, 2006, pp. 459-496.

[17] T. LiU, On lattices in semi-stable representations: a proof of a conjecture of Breuil, Comp. Math., 144, 2008, pp. 61-88.

[18] J. NEKOVAR, Level raising and Selmer groups for Hilbert modular forms of weight two, to appear in Canadian J. Math. (published online), 81 pages.

[19] S. SEN, Continuous Cohomology and p-adic Galois Representations, Invent. Math., 62, 1980, pp. 89-116.

[20] J.-P. Serre, Corps Locaux, Quatrième édition corrigée, Hermann, Paris.

[21] N. WACH, Représentations cristallines de torsion, Comp. Math., 108, 2, 1997, pp. 185240.

Manoscritto pervenuto in redazione il 7 dicembre 2014. 
